\title{
Learning from Generic Language
}

\author{
Michael Henry Tessler ${ }^{1,2} \&$ Noah D. Goodman ${ }^{1}$ \\ ${ }^{1}$ Department of Psychology, Stanford University \\ ${ }^{2}$ Department of Brain and Cognitive Sciences, Massachusetts Institute of Technology
}

\begin{abstract}
Generic language (e.g., Birds have hollow bones) conveys generalizations about categories and is a fundamental and ubiquitous mechanism of learning about the world. How exactly this learning occurs, however, is unclear: Generics exhibit so much flexibility in how they are interpretated that a quantitative theory of how generics update beliefs has not only not been developed or tested, but is often explicitly dismissed. Here, we explore a hypothesis introduced by Tessler and Goodman (2019) that generics update beliefs via an uncertain threshold like a vague quantifier: A category-property generic $(K s F)$ means that the property $\mathrm{F}$ is expected to be relatively widespread in the category $\mathrm{K}$, where what counts as being relatively widespread is a priori uncertain and resolved by consulting one's prior beliefs about the property. We compare this model to a family of alternatives with the same background knowledge but where generics have a fixed, precise meaning; in addition, we formalize a quantitative model of a strictly conceptual-based approach to generics, where generics are a direct connection to conceptual knowledge. Across three experiments in which we both measure and manipulate prior beliefs, we find the uncertain meaning approach to generics to be the best explanation of participants' highly heterogeneous interpretations of novel generic statements. This result taken together with the result that the same model of generic meaning can explain the variability in generic endorsements as shown by Tessler \& Goodman (2019) suggests that generics are an effective medium for faithful transmission of knowledge between interlocutors. This work adds to the growing enterprise of formal, quantitative studies of human understanding of generic language.
\end{abstract}

Keywords: generics; learning; pragmatics; semantics; Bayesian modeling Word count: 13817

\section{Introduction}

Much of what we learn about the world comes not from our own experience but by learning from others, often via language. Language is a powerful medium through which 
to describe that which goes beyond what can be experienced, conveying abstractions and generalizations. Generalizations about categories are often conveyed using generic language (e.g., Birds have hollow bones; Alligators grow to be 10-feet long; Carlson, 1977). Generics are ubiquitous in everyday conversation and child-directed speech (Gelman, Coley, Rosengren, Hartman, \& Pappas, 1998; Gelman, Goetz, Sarnecka, \& Flukes, 2008; Gelman, Taylor, Nguyen, Leaper, \& Bigler, 2004), and learning from generics is thought to be central to the development of conceptual knowledge (Cimpian \& Markman, 2009; Gelman, 2004). More broadly, the language of generalizations is a powerful medium through which to transmit abstract knowledge effectively (Gelman, 2009) and is a plausible key mechanism behind the accumulation of cultural knowledge across generations (Tomasello, 1999, 2016). Understanding how beliefs are updated from generic language is thus an important issue for cognitive science.

One of the most fundamental components of beliefs are the latent probabilities that we use to make predictions about the future (Shepard, 1987). For a standard category-based prediction problem, the relevant metric is the probability that an instance of a category $k$ has a feature $f: P(f \mid k)$, sometimes referred to as the prevalence of the feature in the category. A common intuition with generics is that they "[...] appear to be commonly taken [interpreted] in a rather strong sense, as though the qualifier always had implicitly crept into their interpretation" (p. 172, Abelson \& Kanouse, 1966); in other words, a common intuition is that generics mean all: $P(f \mid k)=1$. Generics allow for exceptions, however (e.g., not all birds fly, yet Birds fly is a true statement), and so the generic must allow for $P(f \mid k)<1$. Some generics seem to convey prevalence levels substantially less than 100\%: Robins lay eggs intuitively conveys that roughly $50 \%$ (the female robins) lay eggs $(P(f \mid k) \approx 0.5)$; Mosquitos carry malaria conveys a low prevalence value (in true fact, $<1 \%$ of mosquitos carry malaria; $P(f \mid k) \approx 0.01)$. Broadly consistent with these intuitions, prior research has found that adults typically rate the prevalence implied by a generic (e.g., Bears like to eat ants) to be very high (most bears like to eat ants; Gelman, Star, \& Flukes, 2002), except when the features could be construed as being accidental, in which case prevalence is rated substantially lower (e.g., Xs have fungus-covered claws $\rightarrow$ some Xs have fungus-covered claws; Cimpian, Brandone, \& Gelman, 2010; see also Khemlani, Leslie, \& Glucksberg, 2009, 2012 for evidence of similarly weak inferences from generics such as Lions eat people).

The fact that generic statements have such variable interpretations in terms of the property prevalence $P(f \mid k)$ has been taken by many in psychology to imply that there is no coherent, quantitative relationship between the meaning of generics and the prevalence of the feature (Leslie, 2008; Cimpian, Brandone, \& Gelman, 2010; Khemlani et al., 2012; Prasada, Khemlani, Leslie, \& Glucksberg, 2013). Instead, theory-building has proceeded with verbal accounts, appealing to notions of kindhood and conceptual structures, whose relation to quantitative data is often underspecified. The fact that generics have variable interpretations, however, does not falsify the notion that there exists a systematic relationship between

Corresponding author: Michael Henry Tessler, 450 Serra Mall, Building 420, Department of Psychology, Room 316, Stanford University, Stanford, CA 94305, USA. E-mail: tessler@mit.edu Present address: Department of Brain and Cognitive Sciences, Building 46, Room 3027, Massachusetts Institute of Technology, 77 Massachusetts Avenue, Cambridge, MA 02139-4307, USA 
generics and prevalence. Generics, like many other aspects of language, may exhibit a sensitivity to context which modulates how they are interpreted; such a context-sensitivity does not in and of itself rule out that generics update beliefs about prevalence in a consistent, predictable way (see Kochari, Van Rooij, \& Schulz, 2020 for a related argument).

Tessler and Goodman (2019) proposed a quantitative account of generics that synthesized and unified this diverse swath of linguistic expressions. Under this model, the core meaning of a generic is a vague quantifier (similar to many or a lot) whose precise meaning is underspecified and must be inferred in context. This context takes the form of shared, conceptually-based prior knowledge about categories and properties. The model was shown to explain truth judgments or endorsements (e.g., is the sentence Mosquitos carry malaria true or false?) with a high-degree of quantitative accuracy. It also provides a yet-untested substantive hypothesis about interpretations, that is, how beliefs should be updated from generic language.

Basic empirical questions about context-sensitive generic interpretation remain unanswered. Previous experimental work on interpretations of generic statements has revealed categorical distinctions (e.g., generics about properties of accidental or disease states are interpreted less strongly than generics about biological properties; Cimpian, Brandone, \& Gelman, 2010), but the extent of the gradience in generic interpretations has never been examined or quantified in an experiment. Furthermore, though intuitively generics can have weak interpretations (e.g., implying that only a minority of the category have the property), the implied prevalence from the weakest of generics in these previous studies was still quite high (e.g., around 70\% in Cimpian, Brandone, \& Gelman, 2010 Expt. 3), leaving open the question of how weak generic interpretations can be. ${ }^{1}$ Our formal modeling predicts gradience in interpretations and that such very weak interpretations can occur when prior knowledge indicates that the property in question is rare within kinds (e.g., the property only manifests under certain circumstances).

This paper is organized as follows. In the first section, we describe the model of generic meaning proposed by Tessler and Goodman (2019) and show how it predicts prevalence interpretations from a generic statement. We additionally describe alternative models that predict prevalence interpretations from different assumptions about the meaning of a generic; we also formalize a model that predicts prevalence by assuming generics serve as a kind of direct conduit to conceptual knowledge. We compare these formal hypotheses in their abilities to predict and provide a parsimonious explanation for generic interpretation data from three experiments, in which we both measure prior beliefs and generic interpretations. In Experiment 1, we test the models on a diverse stimulus set designed to elicit high by-item variability. In Experiment 2, we examine the models in their capacity to explain data from a replication of Cimpian, Brandone, and Gelman (2010) (Implied Prevalence task). In Experiment 3, we test whether prior beliefs about prevalence are causally related to the interpretation of a novel generic by experimentally manipulating prior beliefs. Together, these

\footnotetext{
${ }^{1}$ Similarly, inferences that members of a category have a property (e.g., "Leo is a lion. Thus, Leo eats people.") can be quite weak, though the extant empirical evidence suggests participants still think that the inference is more likely than not (Khemlani et al., 2012). The measurement in this task was a confidence rating on -3 to +3 scale. All reported mean confidence ratings were greater than the midpoint of the scale.
} 
results provide strong quantitative evidence that generics update beliefs about prevalence in a coherent, consistent way as predicted by the model of Tessler and Goodman (2019).

\section{Computational Model}

Generic language conveys generalizations about categories by predicating a property $f$ of a category $k$ (Carlson, 1977; Leslie, 2008). Given that human generalization from observations can be well described using the language of probability (Shepard, 1987) and Bayesian belief-updating with (often structured) prior knowledge (Tenenbaum, Kemp, Griffiths, \& Goodman, 2011), Tessler and Goodman (2019) propose that the meaning of generics - which convey generalizations - can also be formalized using the probability calculus (cf., Cohen, 1999; van Rooij \& Schulz, 2020). Under their model, the literal contribution of a generic sentence is a function on a listener's subjective probability that a future instance of the category $k$ will have the property $f-P(f(x)=$ true $\mid x \in k)$; in the psychological literature on generics, such a probability is often called prevalence, though the term projectibility (N. Goodman, 1955) may also be appropriate (for consistency with the literature, we maintain the term prevalence). For ease of exposition, we denote this probability as $r_{k f}$.

We begin by considering a Bayesian learner trying to determine how strongly to expect a feature $f$ in an unfamiliar category $k$ (i.e., $r_{k f}$ ). For this category, the learner is ignorant about the prevalence (including, presence or absence) of feature $f$ in $k$; in this situation, the best prior knowledge the learner can use to guide their predictions about the prevalence is their knowledge of other similar categories and/or an abstract intuitive theory of the domain in which the feature is relevant (cf., Nisbett, Krantz, Jepson, \& Kunda, 1983). Thus, for learning about an unfamiliar category, we parameterize the prevalence prior to be a distribution across categories of the feature being predicated in the generic statement: $P_{f}\left(r_{k f}\right) .^{2}$

Standard machinery from formal semantics (Montague, 1973) can be used to formalize the literal meaning of a generic as a threshold-function (e.g., $\llbracket g e n \rrbracket[k][f]=r_{k f}>\theta$ ), and Tessler and Goodman (2019) propose the threshold value for a generic is uncertain and sampled from a uniform prior: $P(\theta)=\operatorname{Uniform}(0,1)$. Thus, the model of the literal meaning for a generic is much like a model of a quantifier (e.g., $\llbracket s o m e \rrbracket[k][f]=r_{k f}>0$, $\llbracket$ most $\rrbracket[k][f]=r_{k f}>0.5, \llbracket$ all $\left.\rrbracket[k][f]=r_{k f}=1\right)$, but one in which the threshold is not specified a priori. Incorporating that likelihood function and prior on thresholds into the learner trying to uncover how strongly to expect the feature in the category $r$, they present a Bayesian model of generic interpretation:

$$
P\left(r_{k f}, \theta \mid \llbracket g e n \rrbracket[k][f]\right)=P\left(r_{k f}, \theta \mid r_{k f}>\theta\right) \propto \delta_{r_{k f}>\theta} \cdot P(\theta) \cdot P_{f}\left(r_{k f}\right)
$$

Here, the truth-functional meaning of a generic is a threshold-function mandating that the prevalence $r_{k f}$ is greater than the threshold $\theta$, represented by the Kronecker delta

\footnotetext{
${ }^{2} \mathrm{~A}$ more general notion of the prevalence prior would explicitly take into account knowledge of the category. We return to this point in the discussion.
} 
$\delta_{r_{k f}>\theta}$ that returns 1 when $r_{k f}>\theta$ (i.e., when the utterance is true) and 0 otherwise. $P(\theta)$ is the context-invariant prior distribution over semantic thresholds for the generic sentence and $P_{f}\left(r_{k f}\right)$ is the learner's property-specific prior beliefs about the prevalence. This model is structurally similar to a recently proposed model for gradable adjective interpretation, e.g., tall means height $(x)>\theta$, but $\theta$ is underspecified (Lassiter \& Goodman, 2017). Following Lassiter and Goodman (2017), $P(\theta)$ is assumed to be uniform, and hence $P\left(\theta=\theta^{\prime}\right) \propto 1 ; \forall \theta^{\prime} \in(0,1)$.

To gain intuition for the model behavior, consider a simplified, discrete scenario where prevalence $r_{k f}$ could take on 4 values corresponding to intervals $-\{0.01-0.25,0.26-$ $0.50,0.51-0.75,0.76-1\}$ - and $\theta$ could take 3 values $-\{0.25,0.5,0.75\}$ (Figure 1A, top). Assume that the prevalence prior is uniform (i.e., each of the four levels of prevalence is equally likely a priori). (Recall that the prior on thresholds $P(\theta)$ is always assumed to be uniform.) Then the thehesold semantics for a generic encoded in Eq. 1 states that the prevalence is greater than $\theta$. For $\theta=0.75$, only the highest level of prevalence $r_{k f}=0.76-1$ will be above the threshold. For $\theta=0.5$, both the highest level of prevalence and the second highest $r_{k f} \in\{0.51-0.75,0.76-1\}$ will be above the threshold. For $\theta=0.25$, all but the lowest level of prevalence $-r_{k f} \in\{0.26-0.50,0.51-0.75,0.76-1\}$-will be above the threshold (Figure 1A, middle). To schematically represent the posterior, we count, for each level of prevalence, how many times it lies above a threshold; then, the relative (unnormalized) posterior weights for levels $\{0.01-0.25,0.26-0.50,0.51-0.75,0.76-1\}$ are $\{0,1,2,3\}$, or after normalization $\left\{0, \frac{1}{6}, \frac{1}{3}, \frac{1}{2}\right\}$ (Figure 1A, bottom). We see inferences about prevalence that tilt toward higher values.

The uncertain-threshold generics model produces inference about prevalence that are highly sensitive to prior beliefs about the property $P_{f}\left(r_{k f}\right)$. Prevalence prior distributions that display more structure (e.g., multimodal distributions) receive interpretations that are highly reflective of that underlying structure. This prior-sensitive interpretation is a desirable feature of the model because generics operate over rich intuitive theories about properties and are believed to receive interpretations that are sensitive to those theories (Leslie, 2007; Gelman, Ware, \& Kleinberg, 2010; Cimpian, Gelman, \& Brandone, 2010; Rhodes, Leslie, \& Tworek, 2012; Prasada et al., 2013). For instance, for biological properties like flying (as in Ducks fly), a listener may be assumed to know that such a property manifests in many categories with $0 \%$ prevalence (e.g., $0 \%$ of dogs, rabbits, etc... fly), but is present in other categories in very high proportions (robins, falcons, pterodactyls, etc...); hence, this listener's prior distribution over prevalence would have at least two modes: one around $0 \%$ and another near 100\% (Figure 1B, second row). A reproductive property like laying eggs can manifest in only one sex of a category, which would manifest as a probability distribution with a different bimodal structure, favoring prevalence levels near $0 \%$ and $50 \%$ (Figure 1B, third row). Finally, some properties may be expected to be present in only a minority of the category (e.g., because of a weak causal connection between the property and the kind such as carries malaria; cf., Prasada et al., 2013) which would follow a distribution favoring low prevalence values (Figure 1B, bottom row). ${ }^{3}$ For each, the generic interpretation is unique

\footnotetext{
${ }^{3}$ In this paper, we often parameterize the prior as a mixture of two Beta distributions, which has the feature that prevalence levels close to $0 \%$ are never exactly $0 \%$ but instead may just be very low numbers. Intuitively, this close-to-zero prevalence component of the distribution corresponds to an accidental or transient cause
} 
A

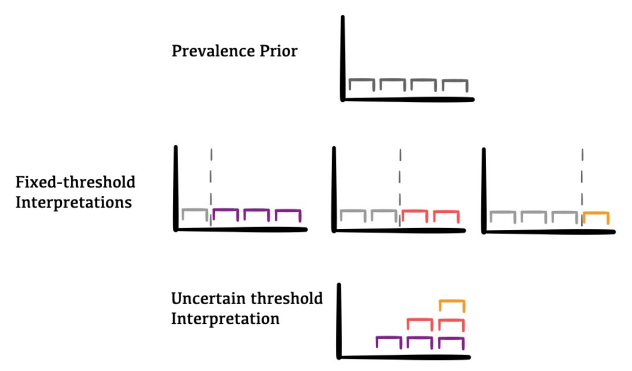

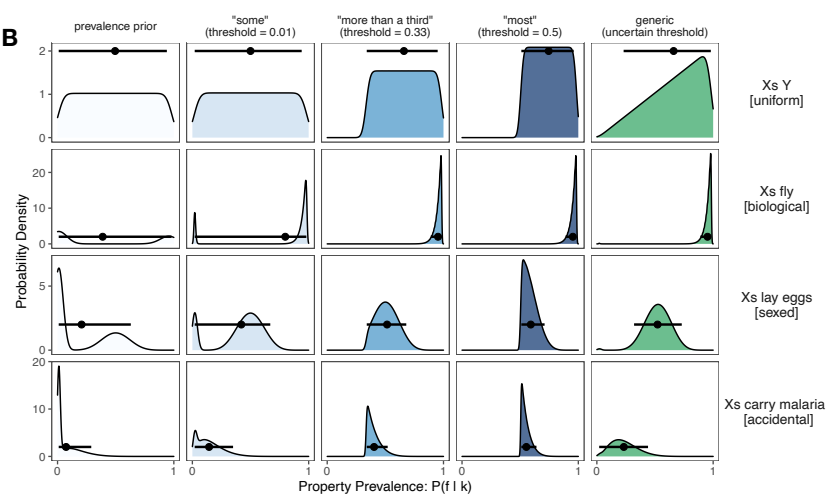

Figure 1. Computational model overview. A: Cartoon of belief updating in the case of a uniform prior over prevalence levels, according to the fixed-threshold and uncertain threshold models. Fixed-threshold models rule out prevalence levels below the threshold (light grey). The uncertain threshold model can be viewed as a composite of all the fixedthreshold models. B: Comparison of uncertain threshold and fixed-threshold models for four different prevalence prior distributions, intuitively corresponding to different types of properties. Priors used for the four properties: uniform $\sim \operatorname{Beta}(1,1)$; biological $\sim 0.6 \times$ $\operatorname{Beta}(1,200)+0.4 \times \operatorname{Beta}(30,1) ;$ sexed $\sim 0.6 \times \operatorname{Beta}(1,200)+0.4 \times \operatorname{Beta}(10,10) ;$ accidental $\sim$ $0.6 \times \operatorname{Beta}(1,200)+0.4 \times \operatorname{Beta}(2,10)$. Lines denote Bayesian $95 \%$ highest density intervals and points denote means of distributions.

(Figure 1B, right-most column) and is most similar to different fixed-threshold quantifiers depending on the property (Figure 1B, other columns).

The formalization of prior beliefs in this model and the vague semantics has been used to explain human truth judgments (or, endorsements) of generics (e.g., that Robins lay eggs is intuitively true, while Robins are female is not) as well as other kinds of linguistic expressions that are thought to convey generalizations (e.g., habituals; Sally runs is less felicitous if Sally ran 3 times in the past year than if she ran 3 times in the past week; Tessler \& Goodman, 2019). Eq. 1 is also mathematically very similar to another recently proposed theory of generics, which was motivated by theoretical constructs from classical conditioning though which also has not yet been tested with quantitative behavioral data (van Rooij \& Schulz, 2020). Here we ask how well belief-updating from generics (i.e., generic interpretations) can be modeled as a function of an uncertain threshold operating over diverse prior beliefs about properties.

\subsection{Alternative models}

We compare our uncertain threshold model to two sets of alternative models, which operate with the same prior beliefs, but which update them according to a different likelihood function.

of the feature, which does not preclude the possibility that the property appears in a few individuals in a category that should not normally have that property present (e.g., a dog that has three legs, an albino raven, ...). Some of the interpretation distributions shown in Figure 1B thus appear bimodal, with the accidental cause component of the distribution appearing with low probability in some posterior distributions. 
2.1.1 Alternative semantics. Many empirical studies in psychology consider a "statistical hypothesis" about the meaning of a generic, which is tantamount to a quantifier with a fixed threshold on prevalence (Prasada \& Dillingham, 2006; Leslie, 2008; Cimpian, Brandone, \& Gelman, 2010; Khemlani et al., 2012; Prasada et al., 2013; Brandone, Gelman, \& Hedglen, 2014). We thus compare the uncertain threshold model to alternative models that update beliefs according to a fixed-threshold semantics (a la quantifiers some or most). To isolate the contribution of the semantics model, we endow the alternative models with the same background knowledge in the form of the prevalence prior distribution $P_{f}\left(r_{k f}\right)$. Models with a fixed semantics are given by:

$$
P\left(r_{k f} \mid r_{k f}>\theta^{*}\right) \propto \delta_{\left[r_{k f}>\theta^{*}\right]} \cdot P_{f}\left(r_{k f}\right)
$$

If $\theta^{*}=0$, then the model is analogous to a literal interpretation for some, which rules out the lowest possible prevalence level $(0 \%) .{ }^{4}$ If $\theta^{*}=0.5$, the model would be analogous to most, with a literal meaning meaning more than half. Other fixed thresholds are possible as well (e.g., $\theta^{*}=0.25$; "more than a quarter"). Predictions of a few of these control models are shown in Figure 1B. We compare our uncertain threshold model to fixed-threshold models with different threshold values, including the best possible threshold that we determine by fitting $\theta^{*}$ to the data.

A model that assumes a fixed threshold of $\theta^{*}$ places zero probability on any response less than $\theta^{*}$. In case there are ratings in the experimental data sets that are less than $\theta^{*}$, we must supplement these models with an extrinsic noise process in order for this model to actually generate predictions for those data; that is, with some probability, we will assume participants respond at random. This noise probability parameter will serve as an additional free parameter for the fixed-threshold control models.

2.1.2 Prevalence prior only. We additionally compare the generic interpretation models to a model that does not update its beliefs from the utterance. This model predicts prevalence ratings according to the prior distribution $P_{f}\left(r_{k f}\right)$. This comparison informs us as to whether or not participants actually update their beliefs when they hear a generic statement, which is an important validation particularly when we manipulate prior beliefs (Expt. 3).

2.1.3 Reified kinds. Conceptual accounts of generics look beyond prevalence to model generic meaning (Leslie, 2008; Cimpian, Brandone, \& Gelman, 2010; Khemlani et al., 2012; Prasada et al., 2013; Csibra \& Shamsudheen, 2015). This approach views conceptual knowledge in the mind as the determining factor of the generic truth conditions (what makes them true or false). For the inverse problem - learning from generic language - these accounts would say that what is learned from a generic statement like Dogs bark is that dogs "are the kind of thing that bark", where the property has some interpretation inside of a conceptual model of the world (e.g., as a generic belief; Prasada, 2000). This approach does not make direct predictions about how implied prevalence should vary as a function of the property being predicated, so we take a step to formalize such a "reified kinds" view of generics.

\footnotetext{
${ }^{4}$ Note that a full model of quantifier interpretation would include their pragmatic interpretations (e.g., that "some" often implies "not all"). Such a model can be implemented in the same probabilistic modeling framework (N. D. Goodman \& Stuhlmüller, 2013) but does not directly address our question of whether a fixed-threshold semantics is tenable for generics.
} 
If there exists a predictable mapping between the meaning " $\mathrm{X}$ is the kind of thing that Ys" and prevalence, then the representation of prevalence (i.e., the prevalence prior) must be able to be partitioned into two clusters: the kinds that do possess $\mathrm{Y}$ and the kinds that do not possess Y. Therefore, the distribution over all kinds will be a mixture distribution of those that have $\mathrm{Y}$ and those that do not have $\mathrm{Y}$. Under such a model, the generic would select the higher mixture component (i.e., the mixture component that has the higher mean prevalence value). For example, if the prevalences from the components are approximated with Beta distributions (as in Figure 1B), the prior would take the following form:

$$
P_{f}\left(r_{k f}\right)=\phi \cdot \operatorname{Beta}\left(\gamma_{1}, \xi_{1}\right)+(1-\phi) \cdot \operatorname{Beta}\left(\gamma_{2}, \xi_{2}\right)
$$

where $\gamma$ represents the mean of the Beta distribution and $\xi$ is the dispersion (or inverse variance). Assume, without lost of generality, that $\gamma_{2}>\gamma_{1}$. Then, the interpretation of the generic in terms of prevalence would simply be:

$$
P\left(r_{k f} \mid u\right) \propto \operatorname{Beta}\left(\gamma_{2}, \xi_{2}\right)
$$

The Reified kinds model is a qualitatively different kind of model than the threshold based models, because Reified kinds relies upon a particular assumption about the structure of prior knowledge (i.e., that it is a mixture distribution). The threshold models are compatible with such a view of the structure of prior knowledge, but they do not require it and would be compatible with other, unstructured views of prior knowledge. The predictions of the Reified kinds model are also only subtly different from those of the Uncertain threshold model. Thus, we turn to a series of quantitative experiments designed with the purpose of teasing apart the models.

\section{Overview of Experiments}

Our models of generic interpretation predict that the interpretations of generics should vary with the prevalence prior for the property in question. Experiment 1 is a large scale version of Cimpian, Brandone, and Gelman (2010)'s implied prevalence task, with sufficiently dense measurements on the by-item level to reveal graded, quantitative variability among interpretations of generics of different properties. Experiment 2 is a replication and extension of Cimpian, Brandone, and Gelman (2010)'s result that implied prevalence varies between generics about biological properties (in particular, body parts with color modifiers e.g., yellow fur) and accidental properties (e.g., fungus-covered claws). Experiment 3 is a test of the causal influence of prevalence priors on generic interpretation, in which we manipulate participants' subjective prevalence priors.

Each of our experiments is divided into two parts. In Part A (Expts. 1a, 2a, 3a), we measure participants' prior beliefs about the prevalence of the features used in our stimuli (e.g., the prior probabilities of different levels of prevalence of the feature likes to cuddle). In Part B (Expts. 1b, 2b, 3b), we measure the prevalence implied by a generic about a novel categories (e.g., Dorbs like to cuddle). We then perform a series of quantitative analyses aimed at predicting the prevalence implied by the generic (Part B) from the prior distribution of prevalence (Part A), assuming different models of belief-updating from a generic (i.e., functions that update a prior distribution into a posterior distribution on prevalence given 
the generic statement). The comparison of different formal models of generic interpretation using the same elicited prior knowledge allows us to provide evidence for a particular model of generic interpretation, while precisely articulating the effect of prior knowledge.

The sample size, exclusion criteria, and planned statistical contrasts for Expts. 1 \& 3 were pre-registered on the Open Science Framework. All cognitive and Bayesian data analytic models were implemented in the probabilistic programming language WebPPL (N. D. Goodman \& Stuhlmüller, 2014). All data, analysis scripts, models, and links to experiments and pre-registration reports can be found at www.github.com/mhtess/ generic-interpretation.

\section{Experiment 1: Variability in Generic Interpretation}

This experiment is designed to test models of generic interpretation by measuring the prevalence implied by a generic about a novel kind, using a diverse set of properties. We first elicit participants' prior beliefs about the likely prevalence levels expected for these properties (Expt. 1a). We then use the elicited prevalence priors to make model predictions about the prevalence implied by a generic (Expt. 1b).

\subsection{Experiment 1a: Prevalence prior elicitation}

In this experiment, we elicit prior beliefs about prevalence for a diverse set of properties. These prior beliefs will be used inside the computational models in order to predict interpretations of generic statements about novel categories. Because the properties are familiar properties, we can elicit participants' knowledge by asking about the property among familiar categories.

\subsubsection{Methods.}

Participants. We recruited 200 participants from MTurk. This number was arrived at with the intention of collecting approximately 23 independent ratings for each property in the experiment; each participant provides 5 ratings per property, so this corresponds to roughly 115 ratings per property. Participants were restricted to those with U.S. IP addresses and with at least a 95\% MTurk work approval rating (these same criteria apply to all experiments reported). The experiment took on average 7 minutes and participants were compensated $\$ 0.80$.

Materials. We created a stimulus set composed of seventy-five properties. Items were generated by the first author by considering eight different classes of properties: physical characteristics (e.g., have brown spots, have four legs), psychological characteristics (e.g., experience emotions), dietary habits (e.g., eat human food), habitat (e.g., live in zoos), disease (e.g., get cancer, carry malaria), reproductive behavior (e.g., have a menstrual cycle), aggressive behaviors (e.g., pound their chests to display dominance, hunt other animals), and other miscellaneous behaviors (e.g., perform in the circus, sing beautiful songs); online sources about strange animal behaviors were consulted in order to find the more obscure properties. Full list of materials can be found in Table A1 in the Appendix.

Procedure and materials. The task was comprised of two different kinds of trials: a category elicitation trial, which appeared first, and twelve prevalence elicitation trials, which used the responses generated in the category elicitation trial. In the category elicitation trial, participants were asked to list three kinds of animals for each of five different classes 
of animals: mammals, fish, birds, insects/bugs, amphibians/reptiles. The five classes of animals were presented in a randomized order on the screen and there were three text boxes for each in which participants could type an animal kind.

On the subsequent prevalence elicitation trials, participants were shown a random subset of five of the animal kinds they generated on the initial trial and asked, for each of these categories, what percentage of the category they believed had a property (e.g., "Out of all of the cheetahs in the world, what percentage do you think attack hikers?"). Participants responded using a slider bar with endpoints labeled $0 \%$ and $100 \%$, and the exact number corresponding to their slider bar rating was displayed once participants clicked on the slider bar. Each participant saw a random selection of twelve properties.

As an attention check, at the end of the prevalence elicitation trials, participants were asked to select, from a list of ten, all of the properties they could remember being tested on. The list included five properties that they had been tested on and five distractors.

4.1.2 Results. We used the same exclusion criteria that were preregistered for the subsequent generic interpretation study: https://osf.io/bwn4t/register/ 5771ca429ad5a1020de2872e. Participants who did not have at least 4 out of 5 hits and at least 4 out of 5 correct rejections during the memory trial were excluded $(n=15)$. In addition, we excluded participants who self-reported a native language other than English $(n=13)$. This left a total of $n=175$ participants, with items receiving on average 28 (range $=[16,38]$ ) sets of participant responses, for a total of on average 140 ratings (range $=[80$, 190]) since each participant provided five ratings.

The prevalence prior distribution can be thought of as describing the expectations about the prevalence of a feature for a novel category that a person has yet to experience. A response in this task can be thought of as a sample from a property's prevalence prior distribution and thus, the distribution of responses for an item are a sample-based approximation of the prevalence prior distribution (Figure 2A). For example, given participants' knowledge of familiar categories, a learner would expect the property has four legs to be either completely universal (prevalence $=100 \%$ ) or completely absent (prevalence $=0 \%$ ) in a novel category. The distribution for the property eats insects looks similar, though there is considerable probability mass spread among the non-binary alternatives $(0 \%<$ prevalence $<100 \%)$. Get in fights with other animals is similar but has substantially less probability mass at $100 \%$ prevalence: It is unlikely that this property is widespread in a category. Live in urban areas shows a monotonically decreasing probability function; even if this property is present in a category, it is unlikely to be highly prevalent. Live in zoos is expected to be even less prevalent, and the property has seizures is expected to even more rare. Some prevalence prior distributions exhibit unique structure; for instance, the property gets erections is only expected to be present in 50\% of the population when it is present at all (presumably because of the fact that this is a property that only male members of categories can possess). In this way, theory-based considerations can influence the structure of these prevalence priors (see also Tessler \& Goodman, 2019 for a discussion of this relationship).

Most of the elicited prevalence prior distributions appear at least bi-modal. To visualize

\footnotetext{
${ }^{5}$ Pilot results indicated similar responses were generated by a question about frequency (e.g., "Out of 100 cheetahs, how many do you think attack hikers?").
} 
all properties simultaneously, we represent each distribution by two statistics: the relative probability mass at $0 \%-P$ (feature is present), or $P(r>0)$ - and the expected value (mean) of the distribution conditional on the prevalence being greater than $0 \%-\mathbb{E}[P(r \mid r>0)]$ or the property's prevalence when present. The $P$ (feature is present) is a measure of the (inverse-)distinctiveness of the feature (i.e., among how many animal categories is the property expected to be present). Prevalence when present is the average prevalence among the categories for which the property can occur. Figure $2 \mathrm{~B}$ shows the distributions of these two parameters: The stimulus set covers a wide range of possible values of both of these parameters. This suggests we have sampled items with priors that exhibit substantial quantitative variability, as desired.

To confirm the multi-modality of the empirical prior distributions, we conducted a Bayesian analysis where we model the prior elicited data (on a by-item basis) as being generated from a mixture of some number of Beta distributions. ${ }^{6}$ We compared the likelihood of the elicited data, modeling each item independently, under a single Beta distribution as well as mixtures of 2-, 3-, and 4- Beta distributions; this comparison allows us to determine the best functional form of the priors, which will be useful when we model the prior data and the generic interpretation data simultaneously, below. The method of model comparison we employ - Bayes Factors - averages across all values of any free parameters of the model (weighted by their prior probabilities) and thus, penalizes models with extra free parameters if, in fact, the model's predictions are not greatly improved by the addition of free parameters (or, are highly sensitive to the exact values of those parameters). Each prevalence prior component (i.e., each Beta distribution) has two parameters governing its shape: a mean $\gamma$ and variance parameter $\xi$; in addition, for models that use mixtures of $2-, 3-$, or 4 -Beta distributions, we have an additional 1, 2, or 3 parameters (respectively) governing the relative contribution (i.e., the weight) of each mixture component. We put uninformative priors over these parameters $\gamma \sim \operatorname{Uniform}(0,1), \xi \sim \operatorname{Exponential}(1), \phi \sim \operatorname{Dirichlet}\left([1]^{n}\right)$ and compute the marginal likelihood of the data by using an Annealed Importance Sampling algorithm (Neal, 2001) implemented in the probabilistic programming language WebPPL. We find that the most parsimonious account of the prior elicited data is that of a mixture of two Beta distributions (log Bayes Factors of 2-vs.1-Beta: $\log \mathrm{BF}_{2,1}=388$; 2- vs. 3-Betas: $\log \mathrm{BF}_{2,3}=178 ; 2$ - vs. 4-Betas: $\left.\log \mathrm{BF}_{2,4}=1094\right)$. Thus, we will assume the prevalence prior data follow a mixture of 2-Beta distributions in analyses below.

\subsection{Experiment 1b: Generic interpretation}

\subsubsection{Methods.}

Participants. We recruited 200 participants from MTurk. This number was arrived at with the intention of getting approximately 50 ratings for each unique item in the experiment. ${ }^{7}$ The experiment took on average 5.50 minutes and participants were compensated $\$ 0.80$.

\footnotetext{
${ }^{6}$ We use Beta distributions to match the form of the response variable: a number between 0 - 1 .

${ }^{7}$ The intended number of ratings in this experiment is smaller than the corresponding number of ratings in the prior elicitation task (Expt. 1a), which was approximately twice as many. In the prior task, we were eliciting multimodal distributions, which require more samples to approximate faithfully than unimodal distributions, which we anticipated for this generic interpretation task.
} 
A

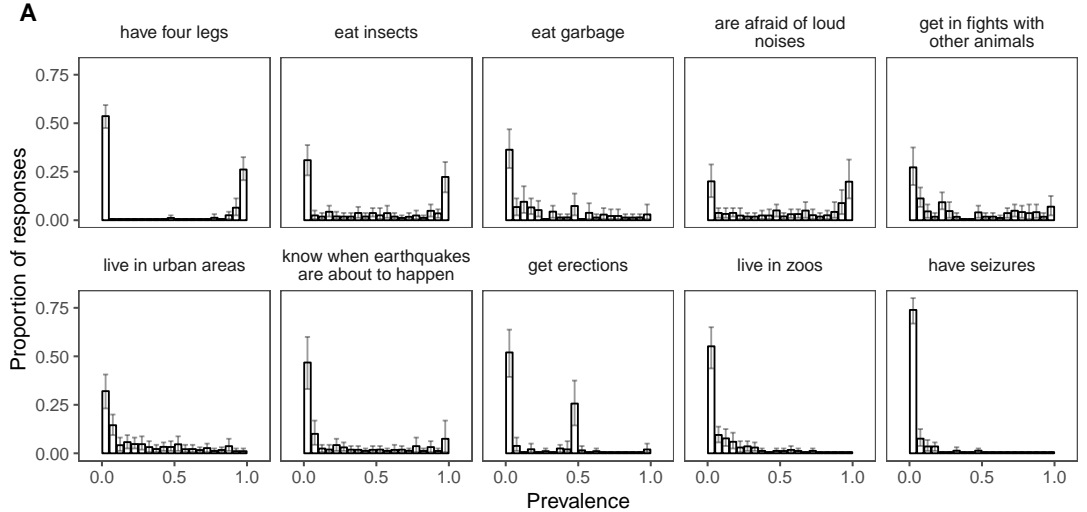

B

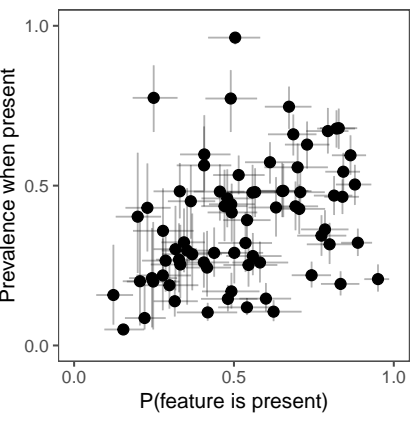

Figure 2. Prevalence priors for a diverse set of animal properties. A: Ten example prevalence priors elicited in Expt. 1a. Distributions vary in terms of how rare the property is across categories (probability mass at $0 \%$ prevalence) as well as the prevalence within a category where the property is present (shape of non- $0 \%$ part of the distribution). B: Prevalence priors summarized by their relative probability mass at zero-prevalence $\mathrm{P}$ (feature is present), and their expected value among non-zero prevalence levels: Prevalence when present. The stimulus set covers a wide range of possible values of both of these parameters. Error bars denote bootstrapped $95 \%$ confidence intervals.

Procedure and materials. The materials were the same as in Expt. 1a. Participants were told that scientists had recently discovered lots of new animals that we did not know existed. On each trial, they would be told facts about the new animals and be asked to translate it into the percentage of that animal to which it applies. On each trial, participants read "You are told: generic", where the generic sentence was a bare plural statement about a familiar property $F$ applying to a novel animal category $K$ (e.g., Javs attack hikers).

Participants were then asked "Out of all of the $K$ s on the planet, what percentage do you think $F$ ?" (e.g., "Out of all of the javs on the planet, what percentage do you think attack hikers?"). Participants responded using a slider bar with endpoints labeled $0 \%$ and $100 \%$, and the exact number corresponding to their slider bar rating was displayed once participants clicked on the slider bar. This kind of explicit question about prevalence has been used to reveal differences in the interpretations of generics vs. quantified statements in adults and young children (Gelman et al., 2002; Brandone et al., 2014) as well as differences in interpretations of generics about different properties (Cimpian, Brandone, \& Gelman, 2010). It is not a measure of unconscious probabilistic expectations, which can often deviate from this kind of conscious probabilistic judgment (e.g., Wu, Meder, Filimon, \& Nelson, 2017). ${ }^{8}$ Though the response measure may encourage some amount of gradience in responses (cf., Armstrong, Gleitman, \& Gleitman, 1983), the fact that the numeric rating appears above the slider bar allows participants to provide an exact rating should they desire.

\footnotetext{
${ }^{8}$ It has been observed, however, that probabilistic information conveyed using perceptual features (e.g., spatial extent) can lead to better understanding of probabilities, as measured by performance on a probabilistic search task (Wu et al., 2017). Thus, our use of a perceptually-grounded slider bar could support participants' estimations of probability and bring more alignment between unconscious probabilistic expectations and participants' reported prevalence values.
} 
Novel animal category names were mostly taken from Cimpian et al. (2010) and similar studies on generic language. Each participant completed thirty-five trials, corresponding to a random subset of the full stimulus set. After the generic interpretation trials, participants completed the same memory check trial as was done in the prior elicitation task (Expt. 1a). They were shown ten properties and asked to click on those they had seen in the experiment. Following the memory check trials, participants completed up to five explanation trials, depending on their ratings in the task. On an explanation trial, participants saw a rating they had given for a property they had rated as applying to less than $50 \%$ and asked if they could explain why they gave the response that they gave. Participants also had the option of changing their response after providing an explanation. These data were only used in exploratory analyses and not for the main analyses reported below. If participants gave no ratings less than $50 \%$, they did not complete any explanation trials.

4.2.2 Descriptive results. We used preregistered exclusion criteria, which were also used for Expt. 1a. Participants who did not have at least 4 out of 5 hits and at least 4 out of 5 correct rejections during the memory trial were excluded $(n=62)$. In addition, we excluded participants who self-reported a native language other than English $(n=8)$. This left a total of $n=132$ participants, with items receiving on average 62 responses (range $=$ $[50,77])$.

We observe a clear gradient in the implied prevalence ratings across our seventy-five items (Figure 3). On one end of the continuum, the generic Wugs have four legs is interpreted as a universal (applying to exactly 100\% of wugs) by half of participants (36 out of 70) and which received a mean implied prevalence rating of 0.96 (95\% confidence interval: $[0.93,0.98]) .{ }^{9}$ On the other end of the spectrum is Glippets perform in the circus, which is interpreted as applying to less than $25 \%$ of glippets by over half of participants (35 out of 61 ) and which receives a mean rating of 0.31 [0.23, 0.39]. Additionally remarkable is the distribution of responses for individual items: Though Feps live in zoos is taken by many participants to mean that around $25 \%$ of feps live in zoos, others take it to mean that almost $100 \%$ live in zoos; such variability in interpretation is not unreasonable: some animal categories (e.g., Micronesian Kingfishers) exist entirely in zoos, while others do not. In addition to the extreme endpoints, we find average ratings at all levels of prevalence in between, even for predicates that have the same main verb (e.g., live in trees vs. live in urban areas vs. live in high-rise buildings vs. live in zoos; compare also properties involving the verb eat; Figure 3), arguing against a view that variability can be traced back simply to verbs with different "generalizing powers" (Abelson \& Kanouse, 1966; Cimpian, Brandone, \& Gelman, 2010). This extreme gradience also stands in contrast to the results of Cimpian, Brandone, and Gelman (2010), which left open the possibility that there were just a few special distinctions of properties that had unique implied prevalences (e.g., biological vs. accidental properties; see also Figure 8). Explaining the extreme gradience we observe in our data set requires a quantitative theory.

To determine whether the variability we observe can be traced back to differences in how individual participants' use the slider bar (e.g., how extreme or moderate a participants is in their usage of the slider bar ratings), we normalized each participant's ratings by

\footnotetext{
${ }^{9}$ Note the novel category term is randomized for each participant and property. We use particular novel category terms in the text for ease of exposition.
} 
subtracting the mean and dividing by the standard deviation of that participant's responses. We then compared the mean normalized ratings by-item to the mean unnormalized (original) ratings by-item. We find an extremely strong correlation between the two $(\mathrm{r}=0.9947)$, which suggests that the gradience in mean ratings we observe by-item is not driven by individuals' variability in how extreme or moderate their responses are.

To assess the reliability of these data, we ran a replication $(n=140)$ using a slightly different dependent measure. ${ }^{10}$ Instead of being asked a question about percentages (e.g., "Out of all of the Ks in the world, what percentage F?"), participants were asked a question in terms of frequency: "Out of $100 \mathrm{Ks}$, how many do you think F?". The empirical byitem means between these data and the original data are highly correlated $(r(74)=0.96$, $\left.r_{\text {spearman }}(74)=0.96\right)$, indicating very high data reliability.

We also see evidence of quantization of responses, especially around $50 \%$ prevalence. The quantization can be observed at $5 \%$ increments and is likely due to the fact that the slider bar displayed the exact percentage above the slider bar and that people prefer round numbers. In our model-based analysis below, we discretize the state space to increments of $5 \%$, and this quantization does not change any of the theoretical conclusions we draw.

4.2.3 Model-based analyses. Our model-based analyses ask how well the various models for the meaning of a generic accommodate the human generic interpretation data and provides the most parsimonious view of the data. We compare our uncertain threshold model to fixed-threshold models for fixed-threshold values between $0 \%$ and $60 \%$ (e.g., fixedthreshold at $0 \%$ is analogous to the quantifier "some"; fixed-threshold at $50 \%$ is analogous to the quantifier "most"). We additionally compare to a "best" fixed-threshold model, where the model's semantic threshold is a global, free parameter inferred from the data. Finally, we include a comparison to a formalization of a "reified kinds" control model, where what the generic does is pick out the component of the prior distribution which has the higher mean level of prevalence. Since fixed-threshold models assign 0-probability to all levels of prevalence below the threshold, these models must be supplemented with an extrinsic noise process (e.g., random guessing). We estimate the noise parameter and all other model parameters using Bayesian methods in order to determine the most likely values of the latent parameters given our hypotheses about how both the prior elicitation and generic interpretation data were generated. ${ }^{11}$ We compare the uncertain threshold model to the fixed-threshold + noise models and the reified kinds model using the standard Bayesian model comparison metric: marginal likelihoods, or Bayes factors (Lee \& Wagenmakers, 2014).

Model implementation details. For each candidate model of generic interpretation (fixed-threshold, reified kinds, and uncertain threshold models), we build a joint

\footnotetext{
${ }^{10}$ By experimenter error, only seventy-four of the seventy-five items were collected in the replication data set.

${ }^{11}$ Appendix C in Tessler and Goodman (2019) provides a careful analysis of the behavior of this joint Bayesian inference strategy for jointly modeling the prevalence prior parameters together with other parameters of the model governing generic interpretation. They find imperceptibly small differences between the parameters inferred from the prevalence prior data in isolation and those inferred by jointly modeling the prior elicitation together and the generic interpretation data, suggesting that in order to provide a stronger quantitative fit to the generic interpretation data, the prevalence priors are not significantly distorted by this method.
} 


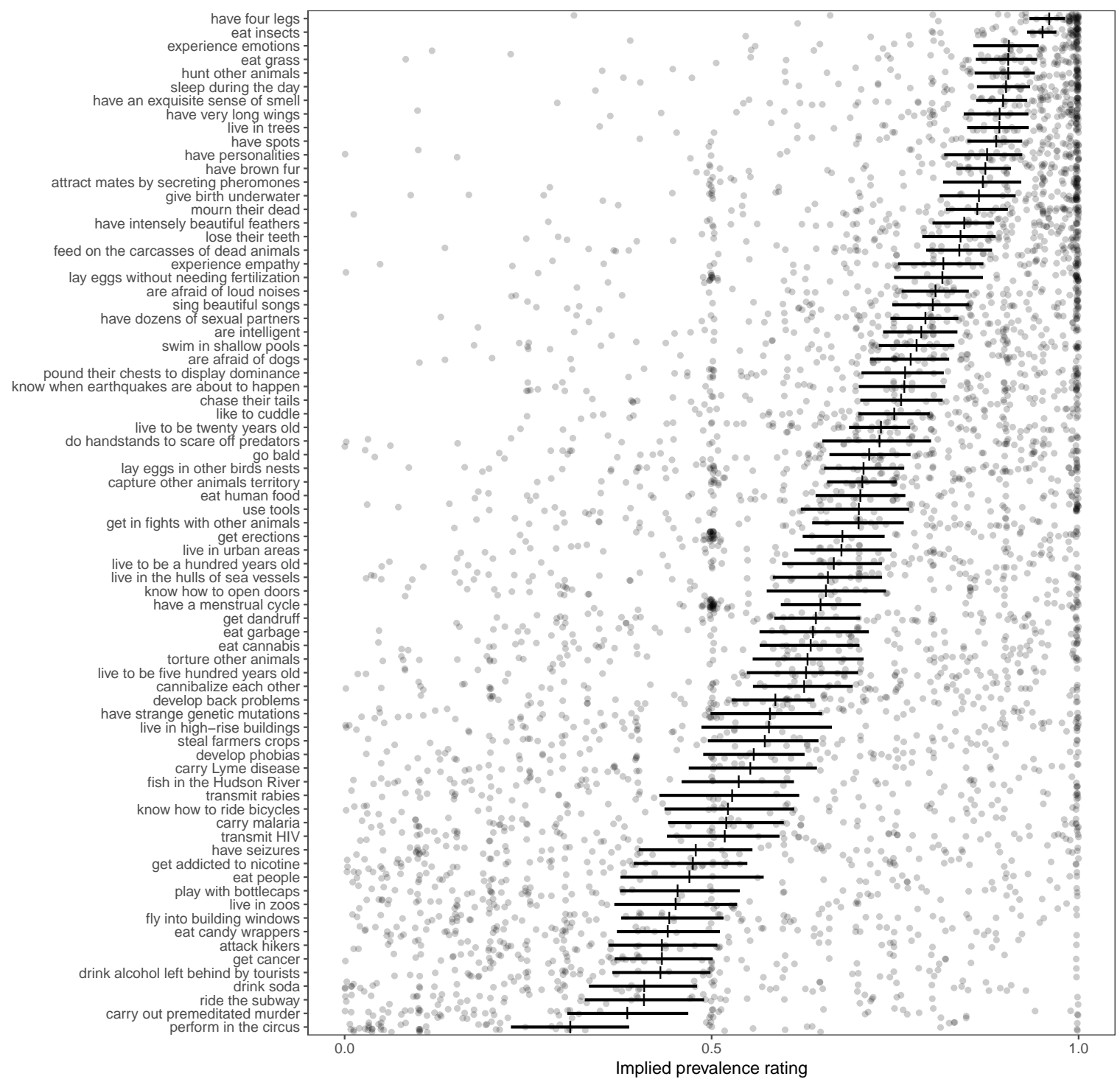

Figure 3. Implied prevalence ratings for generics about novel kinds with seventy-five different properties. Vertical lines denote means, points are individual empirical judgments. Error bars denote bootstrapped $95 \%$ confidence intervals. 


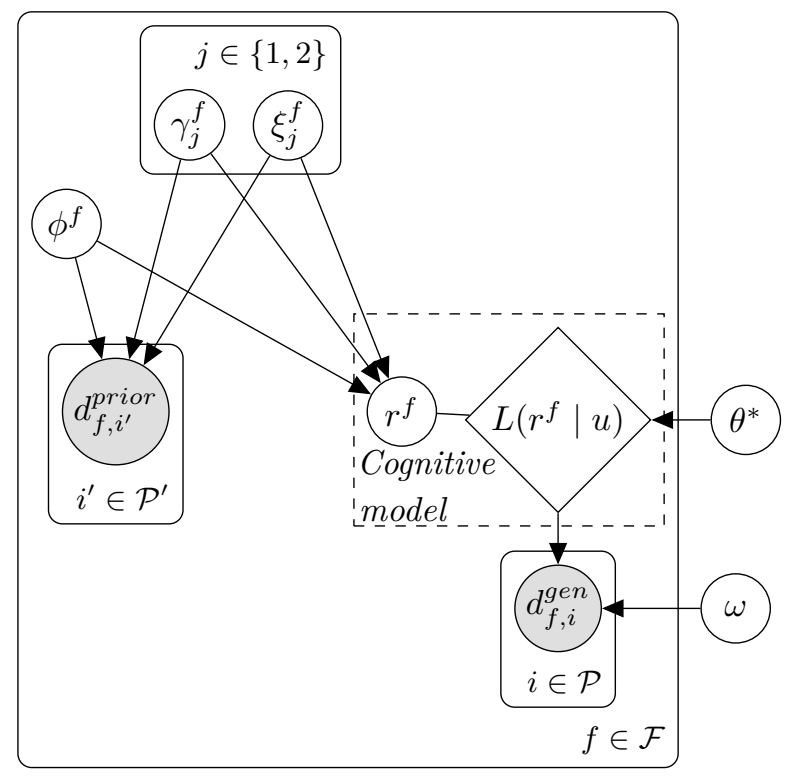

$$
\begin{aligned}
& \omega \sim \operatorname{Uniform}(0,1) \text { [fixed threshold models only] } \\
& \theta^{*} \sim \operatorname{Uniform}(0,1) \text { ["best" threshold model only] } \\
& \phi_{f} \sim \operatorname{Uniform}(0,1) \\
& \gamma_{j}^{f} \sim \operatorname{Uniform}(0,1), \xi_{j}^{f} \sim \operatorname{Exponential}(1) \\
& d_{f, i^{\prime}}^{\text {prior }}, r^{f} \sim \phi^{f} \cdot \operatorname{Beta}\left(\gamma_{1}^{f}, \xi_{1}^{f}\right)+\left(1-\phi^{f}\right) \cdot \operatorname{Beta}\left(\gamma_{2}^{f}, \xi_{2}^{f}\right) \\
& \begin{array}{l}
\text { Uncertain threshold model } \\
L\left(r^{f}, \theta \mid u\right) \propto \delta \llbracket u \rrbracket\left(r^{f}, \theta\right) \cdot P\left(r^{f}\right) \cdot P(\theta) \\
d_{f, i}^{\text {gen }} \sim L\left(r^{f} \mid u\right)=\int_{\theta} L\left(r^{f}, \theta \mid u\right) \mathrm{d} \theta \\
\text { Fixed threshold models } \\
L\left(r^{f} \mid u, \theta^{*}\right) \propto \delta \llbracket u \rrbracket\left(r^{f}, \theta^{*}\right) \cdot P\left(r^{f}\right) \\
d_{f, i}^{\text {gen }} \sim(1-\omega) \cdot L\left(r^{f} \mid u, \theta^{*}\right)+\omega \cdot \text { Uniform }(0,1) \\
\text { Reified kinds model } \\
d_{f, i}^{\text {gen }} \sim L\left(r^{f} \mid u\right) \propto \operatorname{Beta}\left(\gamma_{2}^{f}, \xi_{2}^{f}\right) \text { where } \gamma_{2}^{f}>\gamma_{1}^{f}
\end{array}
\end{aligned}
$$

Figure 4. Fully Bayesian data analytic approach used to model the experimental data. Prior data (Expt. 1a, 2a, 3a) $d^{\text {prior }}$ from each participant $i$ is modeled as a mixture of two Beta distributions, with unknown means $\gamma_{j}$, concentration parameters $\xi_{j}$, and mixture weights $\phi$. The parameters are inferred separately for each feature $f$ (item in Expts. $1 \& 2$, distribution condition in Expt. 3). These parameters determine the prior distribution on prevalence, represented by $r^{f}$, used inside the cognitive models $L$ used to predict the generic interpretation data $d^{\text {gen }}$ from each participant $i$. The cognitive model $L$ is either the uncertain threshold model (Eq. 1), a fixed threshold model (Eq. 2), or the reified kinds model (Eq. 4). Fixed-threshold models are supplemented with an extrinsic global noise process that can be used to generate responses that are below the threshold by randomly guessing. For these models, a noise parameter $\omega$ is used. Finally, a "best" fixed-threshold model is tested where $\theta^{*}$ is a global free parameter inferred from the data.

Bayesian data-analytic model to simultaneously predict the prevalence prior data (Expt. 1a) and the generic interpretation data (Expt. 1b), similar to the methods of Tessler and Goodman (2019) (Figure 4). We model the prevalence prior data as being generated from a mixture of two Beta distributions (as we determined empirically in Expt. 1a) and we model the generic interpretation data as being generated from the candidate computational model of generic interpretation (uncertain threshold, reified kinds, fixed-thresholds + noise). Each prevalence prior thus has five parameters governing its shape: a mean $\gamma$ and variance parameter $\xi$ for each of the two Beta components and a mixture parameter $\phi$ that describe the relative weighting among the two components. We put uninformative priors over these parameters $\gamma \sim \operatorname{Uniform}(0,1), \xi \sim \operatorname{Exponential}(1), \phi \sim \operatorname{Uniform}(0,1) .{ }^{12}$ This model of

\footnotetext{
${ }^{12}$ Another reasonable, less structured model of the prevalence prior data would be a Dirichlet over discretized bins along the prevalence scale. We choose the mixture-of-Betas formulation because it assumes
} 
the prevalence prior $P(r)$, with these parameters, also serves as the prevalence prior in whatever model of generic interpretation we are testing in order to generate predictions for the generic interpretation data. In addition, the models that use a fixed-threshold $\theta^{*}$ semantics for all thresholds $\theta^{*}>0$ have an additional noise parameter, which adds a probability $\omega$ of a participant responding randomly; we put a uniform prior over the value of this noise probability parameter: $\omega \sim \operatorname{Uniform}(0,1)$. The "best" threshold model infers the fixed-threshold $\theta^{*}$ from the data, assuming a uniform prior $\theta^{*} \sim \operatorname{Uniform}(0,1)$. The uncertain threshold model and the reified kinds model have no additional free parameters beyond the prevalence priors.

To learn about the credible values of the parameters as well as generate model predictions for the generic interpretation data, we performed Bayesian inference on each model by running four chains of an incrementalized version of MCMC (Ritchie, Stuhlmüller, \& Goodman, 2016) for $10^{6}$ iterations, removing the first $5 \times 10^{5}$ iterations for burn-in. Convergence was checked qualitatively by confirming the similarity of the results across chains; there were no appreciable differences in the results between different chains. To assess which model is the best explanation (maximally predictive and most parsimonious) of the data, we compute Bayes Factors, which compare the likelihood of the observed data under each of the models, averaging across all values of any free parameters. This form of model comparison penalizes models with extra free parameters if, in fact, the model's predictions are highly sensitive to those parameters. To approximate the marginal likelihood, we used an Annealed Importance Sampling algorithm (Neal, 2001) implemented in the probabilistic programming language WebPPL; for each model, we collected 100 samples, each of which was composed of an annealing schedule of 25,000 linearly spaced iterations. We compare our joint data-analytic models (Figure 4) in their ability to predict the entire distribution of responses for each property in both the prior elicitation task and the generic interpretation task.

Model results. Overall, we find extremely strong evidence that the best model for how generics update beliefs is via an uncertain threshold semantics. The uncertain threshold model is many orders of magnitude better at jointly accounting for the data from the generic interpretation and prior elicitation tasks than any other model (Table 1, log BF).

To gain deeper insight into why the uncertain threshold model is so much better than the alternative models, we examined each model's posterior predictive distribution of responses for each item. The posterior predictive distribution encodes the model's best guesses about what the data should look like, given the model and the parameters inferred from the data. We examined the posterior predictive distribution in two forms: (1) the expected value (or, mean) of the predicted responses for each item, and (2) the full distribution of responses for each item. In order to calculate summary statistics concerned the full distribution of responses, we discretized the model's predicted distribution and the empirical data to 20 equally spaced bins (bin-width $=0.05$ on the $0-1$ scale). With these comparisons, we find that the uncertain threshold model explains roughly $96 \%$ of the variance in the mean data and about $71 \%$ of the variance in the full distributions, with a very small mean squared error in comparison to the alternatives (Table 1).

some correlation between neighboring values along the scale and has fewer parameters. 

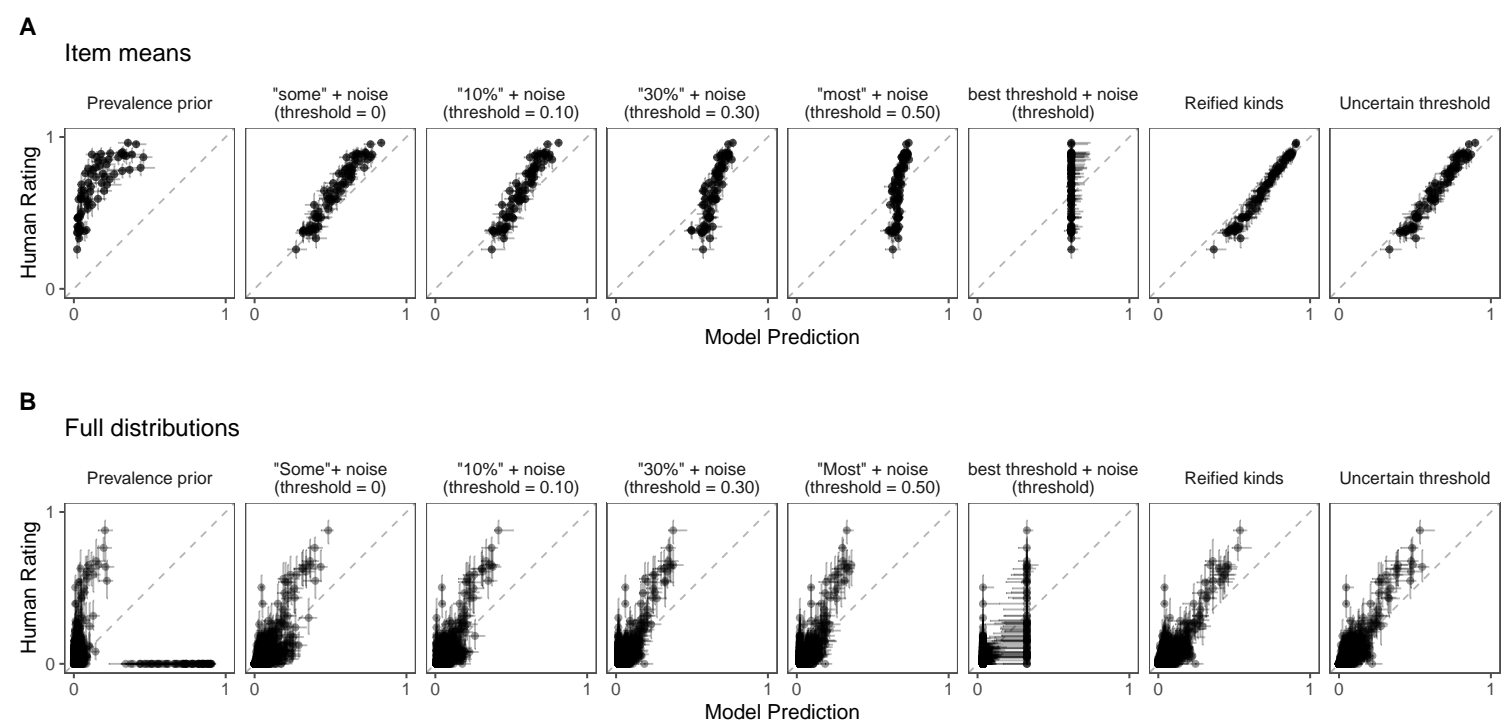

Figure 5. Posterior predictive model fits for eight models in terms of their fit for the interpretations of novel generics. (A) shows the fit to the means of each item and (B) shows the fit to the full distribution of responses for each individual item. Full distribution plots are created by discretizing the distributions to bins of width 0.05 (20 bins per item). Models are (from left to right): (i) the mean of the prevalence prior, (ii - v) a threshold semantics fixed at 0.01 ("some"), $0.1,0.3$, and 0.5 ("most"), (vi) a threshold semantics with a threshold inferred from the data ("best threshold"), (vii) the "reified kinds" model that picks out the component of the prior distribution with a higher mean, and (viii) the uncertain threshold model. Fixed-threshold models are outfitted with a noise parameter to accommodate data points that are logically impossible given the fixed semantics. Error bars denote bootstrapped $95 \%$ confidence intervals for the behavioral data and $95 \%$ highest posterior density intervals for the model predictions.

As a sanity check, we see that the model that predicts the implied prevalence ratings based only on the prevalence prior (Prevalence Prior) does a poor job at predicting the mean human ratings $\left(\mathrm{MSE}=0.27, r^{2}=0.61\right)$, with the model predicting lower prevalence values than participants report, consistent with the generic updating a listener's prior beliefs into stronger posterior beliefs (Figure 5A). Fixed threshold models exhibit an interesting inability to account for the mean implied prevalence ratings. The lowest threshold $(0 \%$ ["some"]) shows a strong correlation with the data, but like the Prior Prevalence model, predicts prevalence ratings that are too low relative to the human data, consistent with the intuition that assigning the semantics of some for generics is too weak. As the threshold value increases, the fixed-threshold models predict higher levels of implied prevalence, leading to model predictions that are too strong for certain generics (model prediction $>>$ human rating); this too-strong interpretation, in turn, leads the data analysis model to infer higher levels of noise as the threshold increases (MAP estimate of noise shown in Table 1). This relationship between threshold and noise is most dramatically exemplified by the "best threshold" model, in which both threshold and noise are inferred from the data: this model 


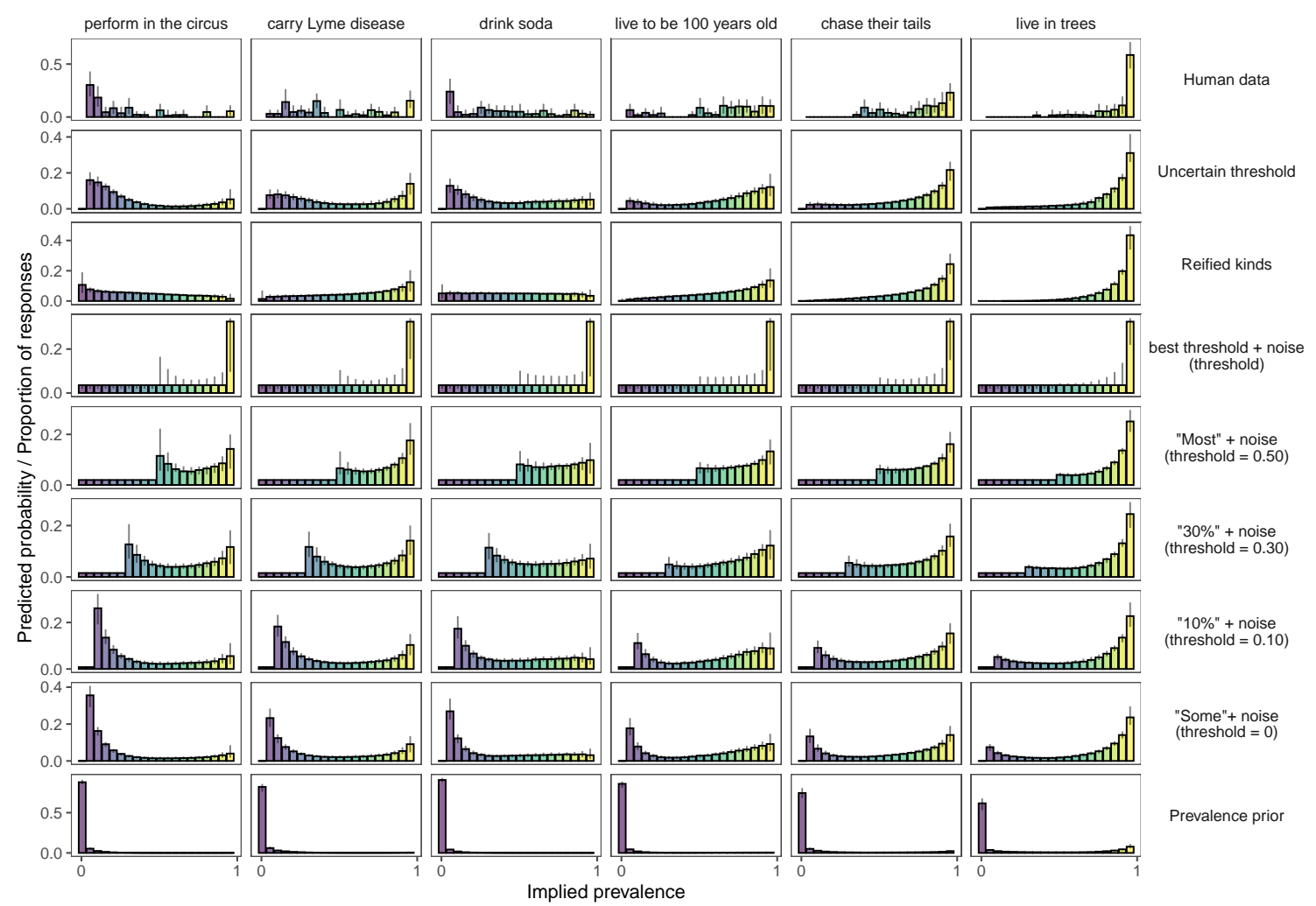

Figure 6. Distributions of interpretations of novel generics in terms of prevalence ratings and model predicted distributions of responses for six items that span the spectrum of prevalence ratings. Individual fixed-thresholds capture the item distributions for certain items, but only the uncertain threshold captures all of the key features of the distributions for all of these items. The Reified kinds model closely tracks the Uncertain threshold model predictions, but comes apart for items where a multimodal distribution of interpretations is predicted (e.g., carry Lyme disease).

ends up deciding that the threshold should be very high (MAP estimate $=0.93$ ) as should the amount of noise (MAP estimate $=0.71)$, resulting in predictions that do not vary considerably by-item. Visually, this relationship between threshold value and noise results in a rotation of the model prediction line; as more responses must be attributed to noise, the model vs. data line appears more like a vertical line (Figure 6).

The alternative model that performs the best is the Reified kinds model, which predicts prevalence ratings based on a categorical (as opposed to continuous) view of the prevalence prior. This model selects the component of the prevalence prior distribution that has the highest mean and uses that to make predictions for generic interpretations (in the case of our 2-component prevalence prior, this model simply selects the component with the higher mean prevalence). This model seems to struggle the most with the "weak generics", the statements that receive overall low prevalence interpretations (Figure 5A; "Reified kinds", items below $y=x$ line). By comparing the distribution of responses for Reified kinds vs. Uncertain threshold, we can see that Reified kinds fails to predict certain 
bi-modalities that the Uncertain threshold model predicts. For example, the statement Feps carry Lyme disease is interpreted by some participants to mean that most or all feps carry Lyme disease, while others interpret it more weakly (some or few feps carry Lyme disease; Figure 6). The Reified kinds model partitions the prevalence prior into two distinct components and always selects the one with higher mean prevalence, thus only predicting the interpretation that most or all feps carry Lyme disease. By contrast, the Uncertain threshold model treats the prevalence prior distribution as a continuous representation of belief about prevalence and is able to predict the bimodal interpretation. Overall, the Uncertain threshold model is able to predict key signatures of full distributions of behavioral data, which no other model is able to adequately describe (Figure 5B).

\begin{tabular}{lllccccc}
\hline Model & Threshold & Noise & $r^{2}$ & MSE & $r_{\text {dist }}^{2}$ & MSE $_{\text {dist }}$ & (log) BF \\
\hline Uncertain threshold & - & - & 0.96 & 0.003 & 0.71 & 0.002 & 0 \\
Prevalence Prior & - & - & 0.61 & 0.27 & $3.84 \cdot 10^{-3}$ & 0.03 & $-6,468$ \\
Some (0\% threshold) & 0.01 & 0 & 0.92 & 0.016 & 0.52 & 0.003 & $-2,435$ \\
10\% threshold & 0.1 & 0.16 & 0.90 & 0.013 & 0.54 & 0.003 & $-2,463$ \\
30\% threshold & 0.3 & 0.31 & 0.74 & 0.018 & 0.57 & 0.003 & $-2,218$ \\
Most (50\% threshold) & 0.5 & 0.39 & 0.54 & 0.026 & 0.50 & 0.004 & $-2,099$ \\
Best fixed-threshold & 0.93 & 0.71 & 0.20 & 0.034 & 0.40 & 0.004 & $-1,817$ \\
Reified kinds & - & - & 0.98 & 0.0045 & 0.66 & 0.002 & $-1,097$ \\
\hline
\end{tabular}

Table 1

Summary statistics and parameters for models of generic interpretation. Threshold value for Best fixed-threshold model is the MAP estimate inferred from the data; noise values are also MAP estimates inferred from the data. Mean Squared Errors and variance explained are calculated both at the level of the item means and over the distribution over responses discretized to bins of width $0.05\left(r_{\text {dist }}^{2}, M S E_{\text {dist }}\right)$ for the generic interpretation data (Expt. 1b). Bayes Factors quantify evidence in support of each model relative to the Uncertain threshold model (in log scale) and are computed over the full distribution of responses for both the prior elicitation and generic interpretation tasks (Expts. 1 a 8 1b). log BF values below 0 indicate evidence in support of the Uncertain threshold model.

\section{Experiment 2: Replication and Extension of Cimpian et al. (2010)}

We use the same model based approach to re-examine the findings of Cimpian et al. (2010). As before, we measure the prevalence priors (Expt. 2a) and use those in combination with the models to predict the generic interpretation data (Expt. 2b), which is a replication and extension of the Cimpian et al. (2010) task.

\subsection{Experiment 2a: Prior elicitation}

We take advantage of the finding in Expt. 1a that the prevalence priors elicited by asking about alternative categories were best modeled as a mixture of two Beta component distributions. We imagine one component governs the creatures that have some causal mechanism that stably gives rise to the property, while the other component is a null distribution, describing those creatures for which the feature could only be generated by an 
unstable, transient cause. Our prior elicitation task is then a structured task, where we ask about particular parameters of this model of the prevalence prior and use Bayesian data analysis to reconstruct the prevalence priors implied by participants' responses.

\subsubsection{Method.}

Participants. We recruited 40 participants over Amazon's crowd-sourcing platform Mechanical Turk (MTurk). We chose this number of participants based on intuition from similar experiments which were designed primarily to test a quantitative model. Participants were restricted to those with US IP addresses and with at least a 95\% MTurk work approval rating. All participants were native English speakers. The experiment took about 5-7 minutes and participants were compensated $\$ 0.75$.

Procedure. Direct elicitation of background knowledge about the properties used in Cimpian et al. (2010) (e.g., by asking about prevalence in familiar categories as did in Expt. 1a) is difficult because many of the properties are fantastical or obscure (e.g., pink teeth, silver legs, fungus-covered fur) which almost no familiar animals categories possess. Therefore, we interrogate participants' latent beliefs about the property by assuming the mixture distribution validated in Expt. 1a - where the prior is composed of a null distribution and a stable cause distribution - and create an elicitation task to measure two aspects of the prior: (1) the relative contribution of the null distribution (e.g., for how many categories is the property expected to be present at all) and (2) the shape of the stable cause distribution, the prevalence among kinds where the property is present (prevalence when present).

Participants were first introduced to a data-collection robot that was tasked with learning about properties of animals. Participants were told the robot randomly selected an animal from its memory to ask the participant about (e.g., The robot says: "We recently discovered animals called feps."). To measure the (inverse-) relative contribution of the null prevalence distribution, the robot asked how likely it was that there was a fep with the property (e.g., "How likely is it that there is a fep that has wings?"), to which participants reported on a scale from unlikely to likely. To measure the shape of the stable cause distribution - prevalence when present - the robot then asked the likely prevalence assuming that at least an instance that had the property (e.g., "Suppose there is a fep that has wings. What percentage of feps do you think have wings?"). Participants completed a practice trial using the property is female to make sure they understood the meanings of these two questions. For example, it is very likely that there is a fep that is female because almost all animals have female members (high $P$ (feature is present)). Additionally, when present, the property is only expected in about $50 \%$ of the category. Participants were provided corrective feedback if they did not answer the question within a range of valid responses.

Materials. We constructed a stimulus set of forty different properties to explore a wide range of a priori beliefs about prevalence. These items make up four categories of properties: body parts (e.g., fur), body parts of a particular color (e.g., yellow fur), body parts described with a vague adjective (e.g., curly fur), and body parts in an accidental or disease state (e.g., wet fur). Pilot testing revealed more variability for items in the accidental category relative to the other types of properties, and so we used twice as many exemplars of accidental properties. We used eight exemplars of each of the three non-accidental properties (parts, colored parts, vague parts), and sixteen exemplars of accidental properties, building on the stimulus set from Cimpian et al. (2010). List of properties is shown in Table A2 of the Appendix. 
5.1.2 Data analysis and results. Question 1 elicits the mixture parameter of a two-component mixture model: $P$ (feature is present). Question 2 elicits the prevalence in a kind where the property is present - a sample from one component of the distribution. These elicited parameters of the priors display a range of possible values Figure 7A. Biological properties are likely to be present and when present, are likely to be widespread (top right corner of scatter plot). More specific properties (either using gradable adjectives or color adjectives) are expected to be slightly less prevalent among the kinds where the property is present, perhaps reflecting the fact that the same kind of animal can come in many different colors or sizes; in addition, gradable properties (e.g., big claws) imply comparison classes (e.g., big for an X) which could further restrict the prevalence. Finally, accidental properties are roughly equally as likely to be present in a category as the color or gradable properties (same $P$ (feature is present)), but are not expected to be as widespread when present in the category (low prevalence when present).

From these two elicitation questions, we can reconstruct the marginal distributions on prevalence, which we have been calling the prevalence priors. We assume that kinds for which the property is absent have prevalence levels sampled from a Beta distribution that heavily favors numbers close to $0: \operatorname{Beta}(\gamma=0.01 ; \xi=100) .{ }^{13}$ With that assumption, the marginal prior distribution on prevalence is given by: ${ }^{14}$

$$
\begin{aligned}
\phi & \sim \operatorname{Beta}\left(\gamma_{1}, \xi_{1}\right) \\
r & \sim \begin{cases}\operatorname{Beta}\left(\gamma_{2}, \xi_{2}\right) & \text { if } \operatorname{Bernoulli}(\phi)=\mathrm{T} \\
\operatorname{Beta}(\gamma=0.01 ; \xi=100) & \text { if } \operatorname{Bernoulli}(\phi)=\mathrm{F}\end{cases}
\end{aligned}
$$

For data analysis, we assume participants' responses to both questions $(i \in$ \{Question 1, Question 2\}) are generated from Beta distributions: $d_{i} \sim \operatorname{Beta}\left(\gamma_{i}, \xi_{i}\right)$, and put uninformative priors over the parameters of each: $\gamma_{i} \sim \operatorname{Uniform}(0,1) ; \xi_{i} \sim \operatorname{Uniform}(0,100)$. To learn about the credible values of the parameters of the model, we ran MCMC on each item independently for 100,000 iterations, discarding the first 50,000 for burn-in.

Figure 7B shows example reconstructed priors for eight properties. Biological properties (biological, vague, and color body parts) have prevalence distributions that are bimodal with peaks at $0 \%$ and near- $100 \%$ prevalence, but differ in their variance around the $100 \%$-mode. By contrast, accidental properties do not have a substantial second mode. This variability in prevalence priors leads the generic interpretation model to predict different prevalence levels implied by the generic.

\subsection{Experiment 2b: Generic interpretation}

\subsubsection{Method.}

\footnotetext{
${ }^{13}$ Note that we use the noncanonical mean $\gamma$ and concentration $\xi$ (or, inverse-variance) parameterization of the Beta distribution rather than the canonical shape (or pseudocount) parameterization for ease of posterior inference. The shape parameterization can be recovered using: $\alpha=\gamma \cdot \xi ; \beta=(1-\gamma) \cdot \xi$.

${ }^{14}$ All modeling results hold when this null distribution is assumed to be an even more left-skewed $\operatorname{Beta}(\gamma=0.001 ; \xi=1000)$ or just a delta-function at zero $\delta_{p=0}$.
} 

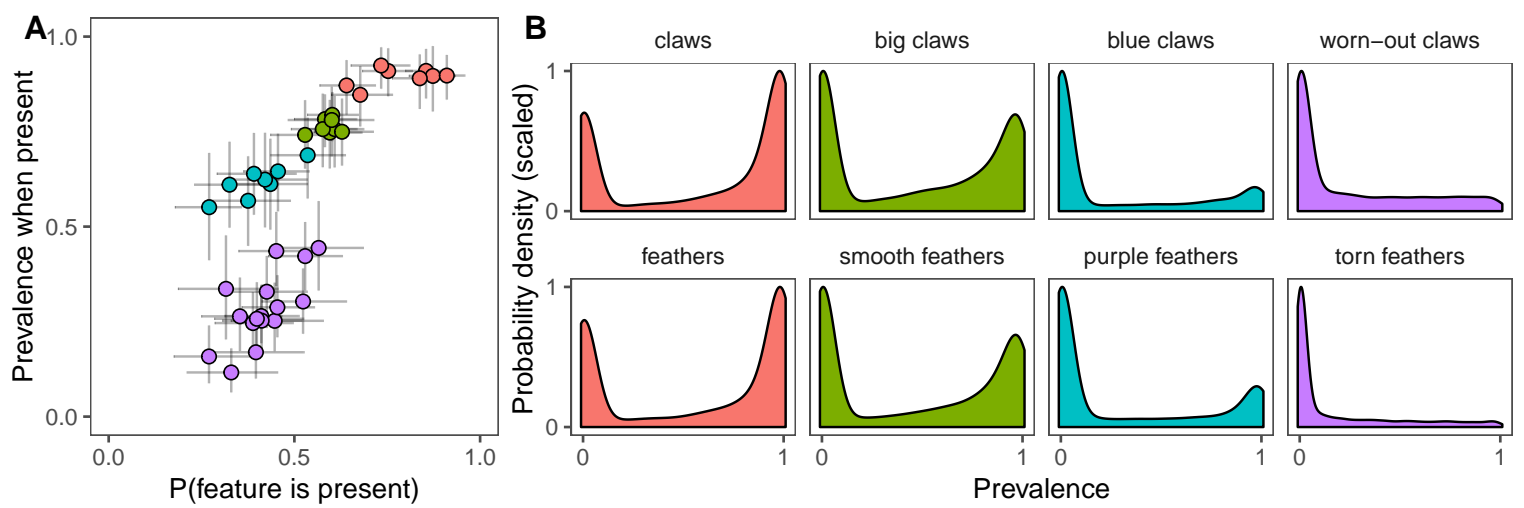

Property type $\square$ body part $\square$ gradable adj + part $\square$ color adj + part $\square$ accidental

Figure 7. Prevalence priors for properties from Cimpian et al. (2010). A: Latent parameters governing prevalence priors are different for different kinds of properties. B: The diversity in parameters gives rise to different underlying distributions over prevalence, which the generic interpretation model uses to make predictions.

A

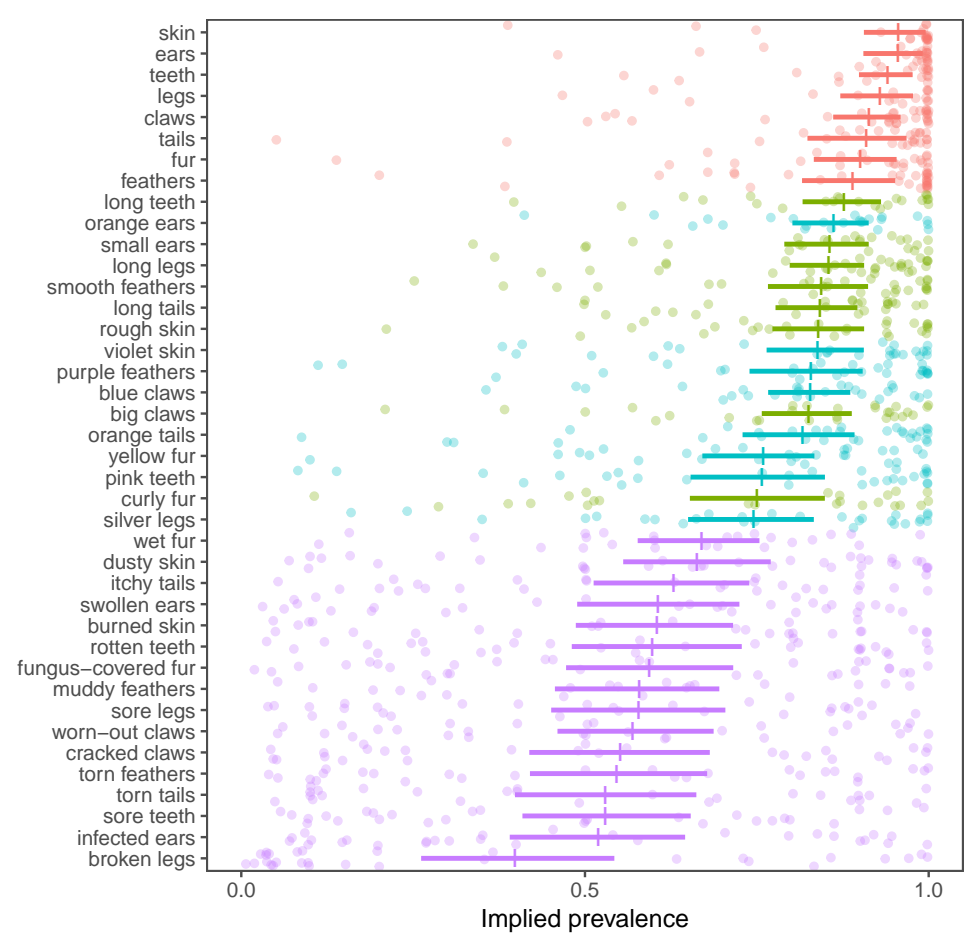

B

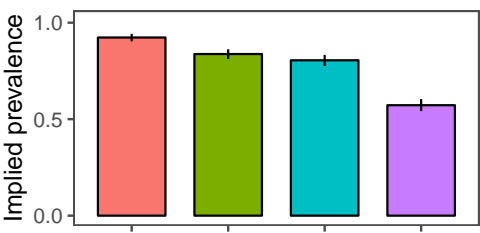

Property type

$\square$ body part

gradable adj + part

color adj + part

accidental

Figure 8. Generic interpretation (implied prevalence) data for items from replication of Cimpian et al. (2010). A: Implied prevalence ratings for forty generics about novel kinds with different properties (Ks have $F$ ). Vertical line denotes mean, horizontal lines denotes bootstrapped $95 \%$ confidence intervals, points are individual responses. B: Implied prevalence collapsed across property type. The original result of Cimpian et al. (2010) showed a difference between color adj + part and accidental. 

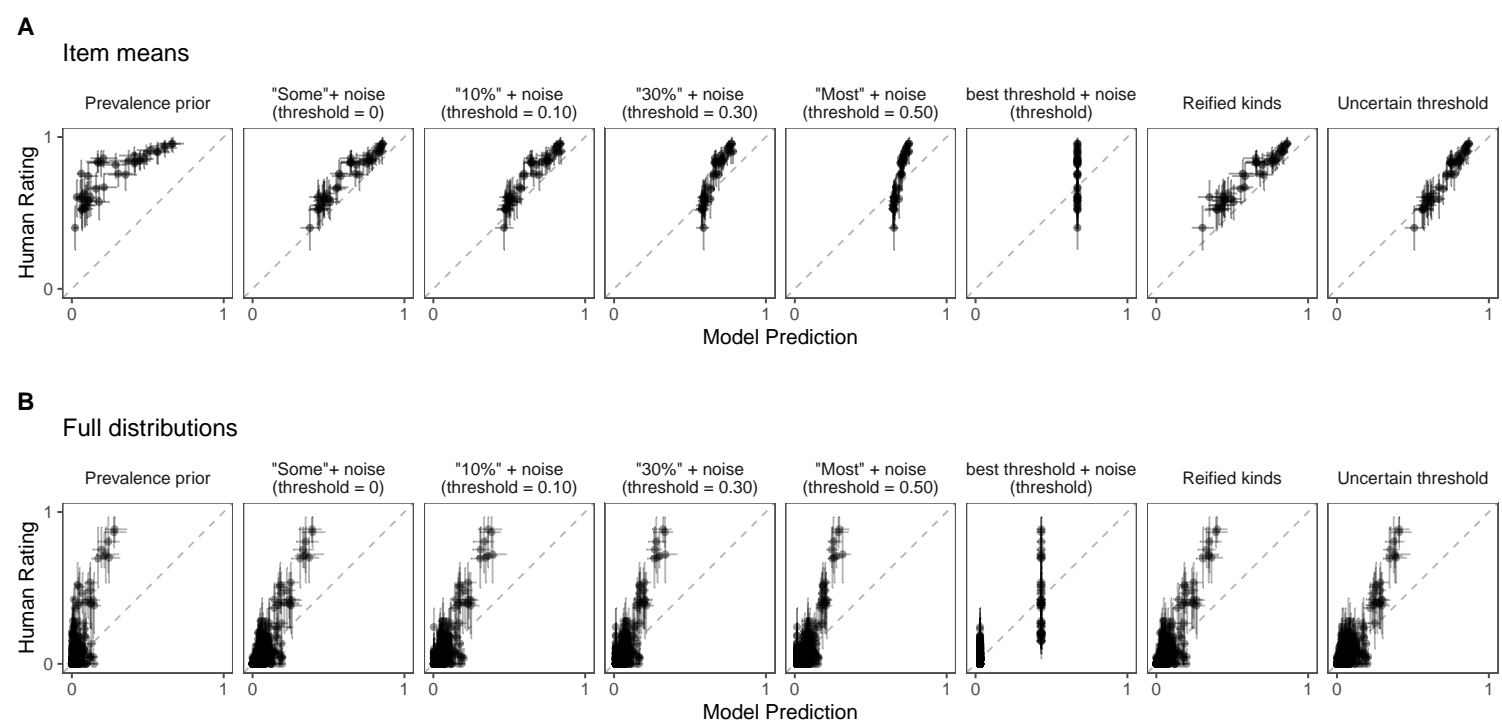

Figure 9. Posterior predictive model fits for the Cimpian et al. (2010) generic interpretation (implied prevalence) replication data. (A) shows item means and (B) full distribution of responses for each individual item. Full distribution plots are created by discretizing the distributions to bins of width 0.05 (20 bins per item). Models are (from left to right): (i) the mean of the prevalence prior, (ii - v) a threshold semantics fixed at 0.01 ("some"), 0.1 , 0.3 , and 0.5 ("most"), (vi) a threshold semantics with a threshold inferred from the data ("best threshold"), (vii) the "reified kinds" model that picks out the component of the prior distribution with a higher mean, and (viii) the uncertain threshold model. Fixed-threshold models are outfitted with a noise parameter to accommodate data points that are logically impossible given the fixed semantics. Error bars denote bootstrapped 95\% confidence intervals for the behavioral data and $95 \%$ highest posterior density intervals for the model predictions.

Participants. We recruited 40 participants over Amazon's crowd-sourcing platform Mechanical Turk. The experimental design is very similar to Cimpian, Brandone, and Gelman (2010), and we chose to have a sample size at least twice as large as the original study (original $\mathrm{n}=15$ ). All participants were native English speakers. The experiment took about 5 minutes and participants were compensated $\$ 0.60$.

Procedure and materials. The properties were the same as in Expt. 2a. Participants were told they were the resident zoologist of a team of scientists on a recently discovered island with many unknown animals and that their task was to provide their expert opinion on questions about these animals. On each trial, participants were supplied with a bare plural sentence of the form $K$ s have $F$, where $\mathrm{K}$ was a novel category label and F was a familiar property (e.g., Feps have yellow fur; see Table A2 in Appendix for list of properties). Participants were then asked to judge prevalence: "What percentage of feps do you think have yellow fur?" Participants responded using a slider bar with endpoints labeled $0 \%$ and $100 \%$, with the exact number corresponding to their slider bar rating displayed once participants clicked on the slider bar. Participants completed in randomized order 25 trials: 5 for each of the biological properties and 10 for the accidental. 


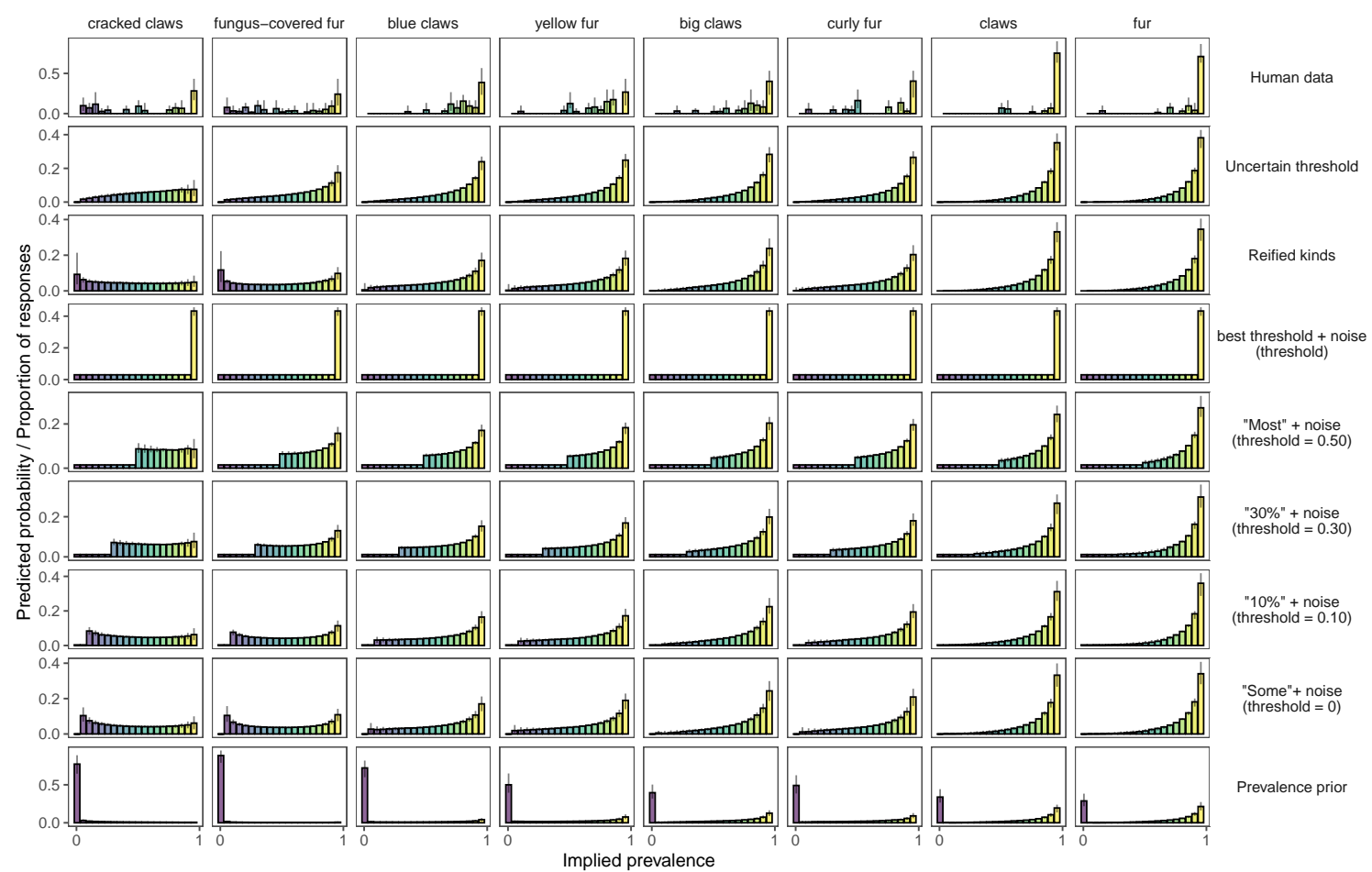

Figure 10. Distributions of implied prevalence ratings and model predicted distributions of responses for eight items from Expt. 2.

5.2.2 Results. Across the forty items in this experiment, we observe a gradient in the implied prevalence ratings similar to that revealed by Expt. 1a. Here, the continuum is mostly clustered by the higher-order type of property (Figure 8A). At the level of property type, Cimpian, Brandone, and Gelman (2010) found a difference between the implied prevalence of generics about biological parts described with color adjectives (e.g., purple feathers) and accidental properties (e.g., fungus-covered claws). We replicate this result and extend it to show further gradability among types of properties (Figure 8B) as well as within types.

Cimpian, Brandone, and Gelman (2010) noted that for the accidental property generics: "properties of this type do not lend themselves very well to generic predication (Gelman, 1988; Cimpian \& Markman, 2008), so generics about broken legs, itchy skin, etc. are infrequent outside the laboratory." (p.1472). It is possible that the order of presentation of the items could influence participants' judgments, with later trials providing more experience to the participant that the experiment is about a particular kind of sentence (i.e., generic statements). ${ }^{15}$ If participants would normally interpret the accidental property generics as existential statements (e.g., Dogs are on my front lawn is classically understood as an indefinite, existential statement and not a generic; Carlson, 1977), then they might provide higher ratings in later trials, as participants gradually impute generic interpretations (and thus, higher prevalence) rather than existential interpretations. Alternatively, over the

\footnotetext{
${ }^{15}$ We thank an anonymous reviewer for suggesting this look at the data.
} 
course of the trials, participants might appreciate more that the property is the informative dimension of variation across trials, and they could provide lower ratings to accidental property generics in the later trials (as a sort of contrast effect). Note that the first explanation is confounded with typical regression-to-the-mean, as items that receive significantly lowerthan-average responses early in the task will tend to receive relatively higher responses later in the task. We investigated this quantitatively by building a Bayesian mixed-effects regression model predicting the response as a function of the property type (biological vs. accidental vs. color vs. vague modifier), the half of the experiment in which the item was rated by the participant, and their interaction. The model additionally included random effects of intercept and effect of split-half by item and intercept, split-half, property type, and their interaction by participant (the maximal random effects structure). We found that participant ratings were slightly higher in the first half than the second half for the accidental items (posterior mean and 95\% Bayesian Credible Intervals: $\beta=0.06[0.03,0.10]$ ), consistent with the latter, dimension of contrast explanation. No difference was observed between first half and second half ratings for the other property type conditions $\left(\beta_{\text {color }}=\right.$ $\left.0.02[-0.03,0.07] ; \beta_{\text {vague }}=0[-0.05,0.05], \beta_{\text {part }}=0[-0.04,0.05]\right)$.

To test our quantitative models in their ability to explain the data from Cimpian et al. (2010), we implemented joint Bayesian data analysis models that predicted the structured elicitation responses (Expt. 2a) and used the inferred prevalence priors to predict the generic interpretation data (Expt. 2b) assuming different models of generic interpretation (uncertain threshold, fixed-thresholds, reified kinds) in a manner directly analogous to what we did for Expt. 1. The priors on parameters were the same as for the modeling of Expt. 1. To compute the posterior distribution over parameters and generate posterior predictive distributions, we ran for each model $3 \mathrm{MCMC}$ chains consisting of $10^{6}$ iterations discarding the first $5 \times 10^{5}$ for burn-in, and checked convergence qualitatively by visual inspection of the parameters and predictions of each chain. We additionally performed a formal Bayesian model comparison by computing the marginal likelihood of the data under each model (in order to compute Bayes Factors); to do this, for each model, we collected 200 samples from an Annealed Importance Sampling algorithm with an annealing schedule consisting of 10,000 uniformly spaced increments.

As we observed in Expt. 1, the best fitting and most parsimonious model of the data from our replication of Cimpian et al. (2010) is the Uncertain threshold model, explaining $96 \%$ of the variance in the mean ratings by item in this data set (Table 2). Both the some model and the Reified kinds model appear to perform similarly well for many items (Figure 9 ), but systematically predict too high of prevalence ratings for items across the scale. Once again, when the data analytic model is allowed to jointly infer the best fixed-threshold and best noise level, the model infers very high levels of noise, washing out any differences one observes across the items (Figure 10). The results show that the differences originally observed by Cimpian et al. (2010) can be accounted for quantitatively by an uncertain threshold model that operates over diverse prior beliefs about properties. 
Table 2

\begin{tabular}{lccccc}
\hline Model & $r^{2}$ & $\mathrm{MSE}$ & $r_{\text {dist }}^{2}$ & $\mathrm{MSE}_{\text {dist }}$ & $(\mathrm{log}) \mathrm{BF}$ \\
\hline Uncertain threshold & 0.96 & 0.0033 & 0.68 & 0.0042 & 0 \\
Prevalence Prior & 0.74 & 0.23 & 0.50 & 0.0083 & -300 \\
Some (0\% threshold) & 0.92 & 0.013 & 0.67 & 0.0048 & -128 \\
10\% threshold & 0.90 & 0.012 & 0.66 & 0.0051 & -199 \\
30\% threshold & 0.87 & 0.014 & 0.59 & 0.0058 & -267 \\
Most (50\% threshold) & 0.89 & 0.017 & 0.53 & 0.006 & -211 \\
Best fixed-threshold & 0.00 & 0.028 & 0.63 & 0.0041 & -55 \\
Reified kinds & 0.93 & 0.015 & 0.68 & 0.0048 & -333 \\
\hline
\end{tabular}

Summary statistics of models for Expt. 2. Mean Squared Errors and variance explained are calculated both at the level of the item means and over the distribution over responses discretized to bins of width $0.05\left(r_{\text {dist }}^{2}, M S E_{\text {dist }}\right)$ for the generic interpretation data (Expt. 2b). Bayes Factors quantify evidence in support of each model relative to the Uncertain threshold model (in log scale) and are computed over the full distribution of responses for both the prior elicitation and generic interpretation tasks (Expts. 2a \& 2b). The Best fixed-threshold model has no variance in its predictions about the item means. $\log B F$ values below 0 indicate evidence in support of the Uncertain threshold model.

\section{Experiment 3: Prior Manipulation}

In our third experiment, we test the causal role of the prevalence prior in generic interpretation. In the previous experiments, participants' prior beliefs about the prevalence of the property - measured by asking about alternative categories (Expt. 1a) or abstract parameters related to the distribution of prevalence across categories (Expt. 2a) - were related to the interpretations of novel generics in terms of prevalence (Expt. 1b, Expt. 2b) via the uncertain threshold model. Though the uncertain threshold model was able to finely track interpretations in a way that alternative models were not, the evidence so far for the influence of the prevalence prior on interpretation is merely correlational. In this experiment, we test the causal influence of the prevalence prior on generic interpretations by manipulating the prior. We first elicit judgments about prevalences following our prior manipulation procedure (Expt. 3a); in addition to serving as a manipulation check, these predictions also serve as the measurements of the (manipulated) prevalence priors used in our models to predict the generic interpretations (Expt. 3b).

\subsection{Experiment 3a: Prior elicitation (manipulation check)}

In this experiment, we elicit participants' beliefs about the prevalence of a property among different categories following a manipulation designed to alter participants' beliefs about a property.

\subsubsection{Methods.}

Participants. We recruited 450 participants from MTurk. This number was arrived at with the intention of getting approximately 40 participants to provide ratings for each unique condition in the experiment, for an intended total of approximately 200 ratings per 
condition. ${ }^{16}$ The experiment took on average 3 minutes and participants were compensated $\$ 0.30$.

Materials. The goal of this experiment was to manipulate the prevalence prior. Participants were familiarized with one of ten prevalence prior distributions, shown in Figure 11E. In Experiment 1a, we found that the prevalence priors for familiar properties were best-modeled as a mixture of two Beta distributions. Hence, in these experiments, nine of our ten distributions were bimodal, with modes either at $0 \%, 25 \%, 50 \%, 75 \%$, or $100 \%$; the tenth distribution was uniform over all prevalence levels between $0 \%$ and $100 \%$. For the bimodal distributions, the mixture between the two modes was always $50 \%$ (half of samples were from one mode and half from the other, as shown in Figure 11B).

Our cover story involved learning about a single property, so that we could assign credit of differences in interpretations back to the manipulated prior distribution, rather than knowledge about properties (which we studied in Experiments 1 and 2). Pilot testing revealed that using a completely novel property (e.g., daxing) made our task highly artificial. Thus, we used a property from Experiment 1 that we thought would be particularly amenable to manipulation by selecting a property whose prevalence prior had high entropy (i.e., participants had lots of uncertainty as to what prevalence levels to expect for this property); the property we used was the predicate know when earthquakes are about to happen.

Procedure. In order to avoid participants learning and reasoning about the structure of the task, participants completed only a single trial: Each participant saw only one prevalence distribution. Participants were given a cover story in which they were asked to imagine they were an astronaut-scientist exploring a distant planet with many new animals on it. They were studying these animals with another scientist to understand the animals' ability to "know when earthquakes are about to happen". Participants were then shown data that they and their fellow scientist had collected about the prevalence of the property among different novel animals. In order to minimize distraction, novel animals were simply labeled by a letter (e.g., "Animal C" or just "Cs"). Information about the animal appeared in an evidence statement (e.g., "After studying the animal, you and your partner determine that $98 \%$ of Cs know when earthquakes are about to happen.") and was displayed in a table showing the corresponding numerical data (Figure 11A). Participants proceeded in a self-paced way by clicking a button to reveal information about the next animal. After participants viewed the data for ten categories, they were told to review the information about the animals before continuing (Figure 11B). Then, the data table was removed and participants were provided the following prompt:

Tomorrow, you and your partner will study 5 other new species of animals. What percentage of each do you think will know when earthquakes are about to happen?

Participants were given five slider bars ranging from $0 \%-100 \%$, and asked to predict the prevalence for the next five categories (Animals K, L, M, N, and O; Figure 11C). After rating the slider bars, we asked participants to explain why they gave the responses that

\footnotetext{
${ }^{16}$ The intended number of ratings is larger than the corresponding number of ratings in the other prior elicitation experiments (Expts. 1a, 2a). We decided to increase the target number of ratings because we anticipated more noise in this task (as a result of it being a single trial experiment) and because we had fewer numbers of unique items or conditions in Expt. 3 than in Expt. 1.
} 
Prevalence prior manipulation (both experiments)

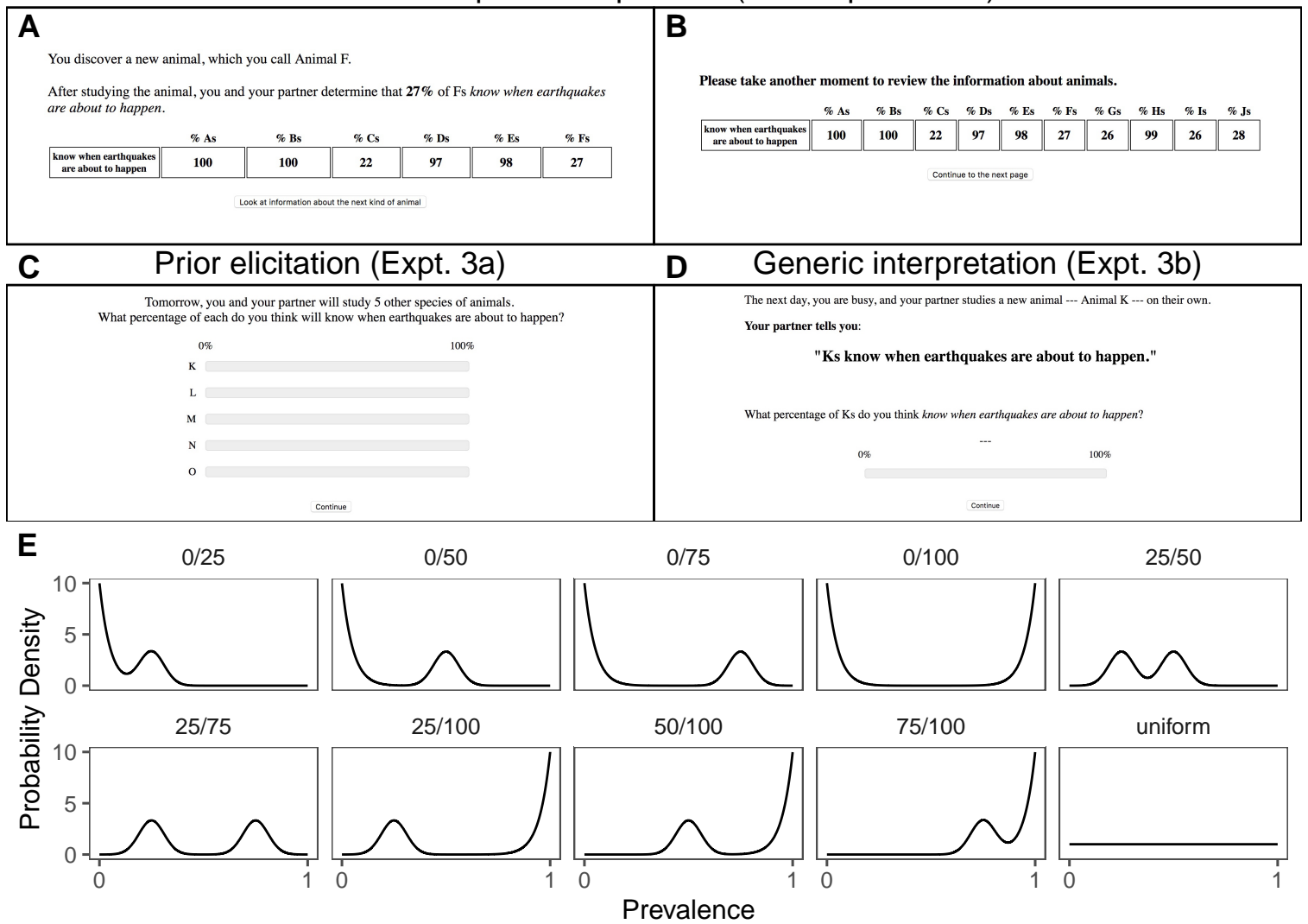

Figure 11. Overview of Experiment 3. A: Prevalences of feature in animals are shown one at a time, described in text and displayed in a table. B: Participants are asked to review previous results once all are displayed. C: Prior elicitation task: Participants predict the results of the next five animals. D: Generic interpretation task: Participants rate prevalence after reading generic sentence about novel category (e.g., Ks know when earthquakes are about to happen). E: Experimentally manipulated prevalence prior distributions. The distribution shown in panels $\mathrm{A}$ and $\mathrm{B}$ is the 25/100 distribution.

they did. Then, they completed an attention check survey where they were asked what property was being investigated (choosing a response from a list of 10 options) and to input one of the prevalence levels they saw on the familiarization screen.

6.1.2 Results. Participants who failed to either select the correct property or list a correct number from the familiarization period were excluded $(n=26)$, in addition to participants who reported a native language other than English $(n=24)$, leaving a total of $n=403$ participants whose data we analyzed. Similar to the data from Expt. 1a, a response in this task can be thought of as a sample from a prevalence prior distribution and the distribution of responses as estimates for the whole distribution. We visualize the distributions by discretizing the responses so that each response goes into one of twenty equally spaced bins (effectively turning the 101-pt scale into a 20-pt scale). Figure 12A (top row) shows the empirically elicited predicted prevalence distributions. These distributions clearly reflect the familiarization distributions supplied to participants in the experiment, 
indicating a successful manipulation, with some idiosyncratic features. The distribution that is most different from the familiarization distribution is the uniform distribution; the empirical distribution is more unimodal with a peak at 50\%. Many distributions exhibit a regression to the mean in some participants' predictions: In the rare or deterministic distribution (which featured prevalence levels either around $0 \%$ or $100 \%$ ), some participants guess that the next animal will have $50 \%$ prevalence; a similar phenomenon can be observed in the weak or strong distribution $(25 \%$ or $75 \%)$ and to a lesser extent in the other bimodal distributions.

\subsection{Experiment 3b: Generic interpretation}

In this experiment, we measure participants' interpretations of generic statements about novel categories following the same manipulation of Expt. 3a, which altered participants' beliefs about the prevalence of a property among different categories (i.e., the prevalence prior).

6.2.1 Methods. The sample size, exclusion criteria, and planned statistical contrasts were all preregistered: https://osf.io/n342q/register/ 5771ca429ad5a1020de2872e.

Participants. We recruited 600 participants from MTurk. This number was arrived at with the intention of getting approximately 50 ratings for each unique condition in the experiment. The experiment took on average 2.70 minutes and participants were compensated $\$ 0.30$.

Materials and procedure. The materials and procedure were almost identical to those of Expt. 3a. The only difference was that following the familiarization period, the data table was removed and participants were provided the following prompt:

The next day, you are busy and your partner studies a new animal on their own:

Animal K. Your partner tells you: "Ks know when earthquakes are about to happen."

In order to encourage participants to pay attention to the language, this text appeared on the screen for five seconds before participants were asked: "What percentage of Ks do you think know when earthquakes are about to happen?" and provided with a slider bar ranging from 0\%-100\% (Figure 11D). As in Expt. 3a, participants then completed an attention check survey where they were asked what property was being investigated and to input one of the prevalence levels they saw on the familiarization screen.

6.2.2 Results. We used preregistered exclusion criteria, which were also used for Expt. 3a. Participants who failed to either select the correct property or list a correct number from the familiarization period were excluded $(n=39)$. In addition, we excluded participants who self-reported a native language other than English $(n=33)$. Finally, we employed one exclusion criterion which was not pre-registered: Participants who responded $0 \%$ to the generic interpretation task ( $n=18 ; 3 \%$ of participants); this response is literally impossible under all models of generic interpretation and indicates a misunderstanding of the task. A post-hoc analysis of these participants' free-form explanations revealed that the majority of these respondents believed they were responding to a prior elicitation question (predict the next animal); it is likely that these participants were not attending to the generic 


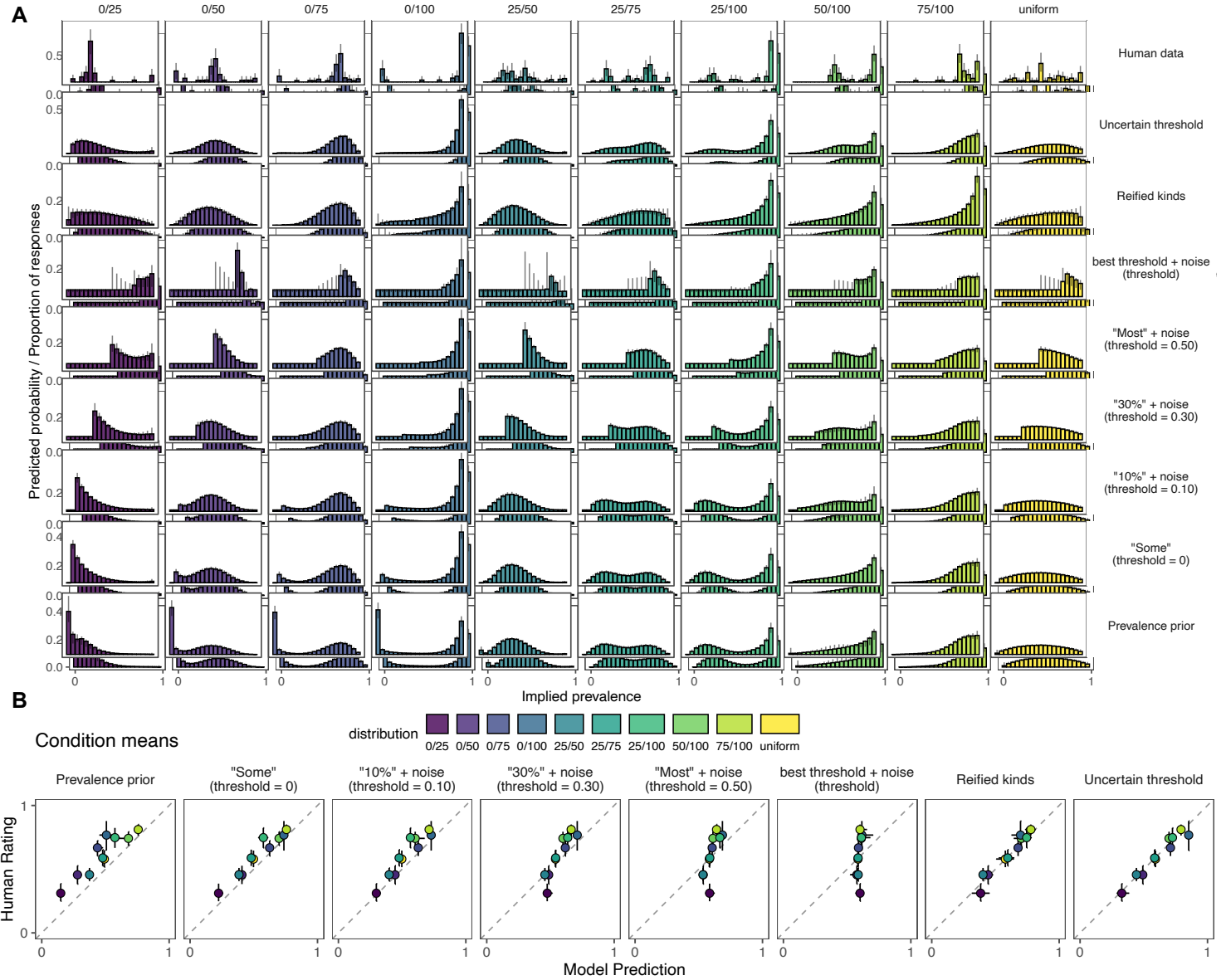

Figure 12. Experiment 3 results. A: Empirical distribution of responses for the generic interpretation task (Expt. 3b; top row), uncertain threshold model predictions, and alternative model predictions. Data and model predictions are shown for ten different prior distribution conditions (horizontal facets). B: Posterior predictive expected values for the quantitative models in comparison to the human empirical means. Error bars denote bootstrapped $95 \%$ confidence intervals for the behavioral data and $95 \%$ highest posterior density intervals for the model predictions. 
statement when it was on the screen. These exclusions left a total of $n=514$ participants, with items receiving on average 51 responses (range $=[40,62]$ ).

Our main qualitative hypothesis is that the same generic sentence (Ks know when earthquakes are about to happen.) would be interpreted differently across conditions. The differences in interpretations are visually evident in the empirical distributions of responses (Figure 12A, top row). More specifically, we predict that the prevalence implied by the generic sentence will, to a first approximation, track the mean of the prevalence prior distribution. To test this, we ran a linear model predicting responses with a fixed effect of the prior mean (as estimated by the data from Expt. 3a). There was a significant main effect of prior mean, with a coefficient close to $1\left(\beta_{1}=0.94, S E=0.07, t=12.76\right)$, indicating a strong linear relationship between the mean of the learned prevalence prior and the resulting interpretations (Figure 12B, left-most facet). Furthermore, there was a significant main effect of intercept, indicating that participants' responses were significantly greater than the mean of the corresponding prevalence priors $\left(\beta_{0}=0.16, S E=0.04, t=4.44\right)$. To more directly test that the distributions of responses in this generic interpretation task (Expt. $3 \mathrm{~b}$ ) were indeed different from the distributions elicited in the prior task (Expt. 3a), we ran exploratory non-parametric Kolmogorov-Smirnoff tests to test for the dissimilarity of the prior and interpretation distributions. We found that eight of ten generic interpretation distributions were significantly different than the prior distributions (Table 3). The two which were not significantly different between prior and generic interpretation were the 50/100 and 75/100 distributions. These distributions are also predicted by the uncertain threshold model to be most similar between prior and posterior; since the prior beliefs about prevalence are so strongly skewed towards high prevalence values, the updating power of a generic is more limited. In summary, these results demonstrate that participants interpret the novel generic sentence in a manner sensitive to the background distribution and that the generic utterance updates participants' beliefs to indicate a prevalence significantly higher than the prior.

We now rigorously test the uncertain threshold model as a theory of belief updating from generics by asking how it serves the two purposes of a psychological theory: prediction and explanation (Shmueli, 2010; Yarkoni \& Westfall, 2017). To test the predictive power of the uncertain threshold model, we can use it and the prior elicitation data (Expt. 3a) to make a number of parameter-free, a priori predictions about quantitative differences in mean implied prevalence ratings across the ten distribution conditions. These predictions were derived by bootstrapping the prevalence prior data to construct bootstrapped $95 \%$ confidence intervals for the uncertain threshold model prediction; from this procedure, we predicted that two conditions should have different mean prevalence ratings if the bootstrapped $95 \%$ confidence interval of the model's predictions did not overlap one another. With those decision rules in mind, we preregistered the following contrast predictions among mean implied prevalences (conditions within \{\} s indicate a prediction of no difference): 0/25 $<\{25 / 50,0 / 50\}<\{25 / 75$, uniform $\}<25 / 75<\{25 / 100,50 / 100\}<\{75 / 100,0 / 100\}$ (predictions also shown in Table 4). To evaluate these predictions, we built a Bayesian regression model with the relevant contrasts using a zero- and one-inflated Beta linking 


\begin{tabular}{lcc}
\hline Distribution Condition & $\mathrm{D}$ & $p$-value \\
\hline $0 / 25$ & 0.52 & $1.01 \cdot 10^{-9}$ \\
$0 / 50$ & 0.31 & $8.94 \cdot 10^{-4}$ \\
$0 / 75$ & 0.34 & $6.43 \cdot 10^{-5}$ \\
$0 / 100$ & 0.31 & $1.77 \cdot 10^{-3}$ \\
$\cdots 25 / 50$ & 0.23 & $1.39 \cdot 10^{-2}$ \\
$25 / 75$ & 0.32 & $3.42 \cdot 10^{-4}$ \\
$25 / 100$ & 0.29 & $1.19 \cdot 10^{-3}$ \\
$50 / 100$ & 0.17 & 0.14 \\
$75 / 100$ & 0.22 & $6.81 \cdot 10^{-2}$ \\
uniform & 0.25 & $8.52 \cdot 10^{-3}$ \\
\hline
\end{tabular}

Table 3

Kolmogorov-Smirnoff test results testing for the dissimilarity between the priors (Expt. 3a) and the generic interpretation (posterior) response distributions (Expt. 3b). The only distributions to be not significantly different between the prior and posterior were the 50/100 and 75/100 distributions; the uncertain threshold model predicts only subtle shifts for these distributions.

function. The maximum a posteriori estimates and $95 \%$ credible intervals of the coefficients of the regression model, as well as the model predicted qualitative differences, are shown in Table 4. Each of the five predicted positive differences were estimated to be greater than zero, and each of the four predicted null differences were not credibly different from zero. These results indicate that the uncertain threshold model serves as a powerful predictive psychological theory.

\begin{tabular}{llrl}
\hline Coefficient & Prediction & Estimate & Credible Interval \\
\hline Intercept & & 0.32 & {$[0.221,0.418]$} \\
$0 / 25$ vs. $\{25 / 50,0 / 50\}$ & $>$ & 0.15 & {$[0.036,0.278]$} \\
$25 / 50$ vs. $0 / 50$ & $=$ & -0.07 & {$[-0.270,0.134]$} \\
$\{0 / 25,25 / 50,0 / 50\}$ vs. $\{25 / 75$, uniform $\}$ & $>$ & 0.34 & {$[0.265,0.409]$} \\
$25 / 75$ vs. uniform & $=$ & 0 & {$[-0.204,0.189]$} \\
$\{25 / 75$, uniform $\}$ vs. $0 / 75$ & $>$ & 1.14 & {$[0.903,1.375]$} \\
$0 / 75$ vs. $\{25 / 100,50 / 100\}$ & $>$ & 1.11 & {$[0.866,1.352]$} \\
$25 / 50$ vs. $50 / 100$ & $=$ & -0.09 & {$[-0.297,0.127]$} \\
$\{25 / 100,50 / 100\}$ vs. $\{75 / 100,0 / 100\}$ & $>$ & 0.52 & {$[0.307,0.743]$} \\
$75 / 100$ vs. $0 / 100$ & $=$ & -0.21 & {$[-0.458,0.035]$} \\
\hline
\end{tabular}

Table 4

Regression model fits for planned comparisons. Predictions are based on whether the 95\% bootstrapped confidence intervals for the uncertain threshold model prediction overlap.

To evaluate the uncertain threshold model as an explanation of the data (and in comparison to its alternatives), we constructed a Bayesian data analysis model to jointly predict the prevalence prior data (Expt. 3a) and the generic interpretation data (Expt. 3b). As we did for Experiment 1, we model the prevalence prior data for each distribution condition as a mixture of two Beta distributions (with unknown means, variances, and mixture parameters) and we model the generic interpretation via the uncertain threshold 
model (or an alternative model) that assumes the prevalence prior inferred from the Expt. 3a data (Figure 4). As before, fixed-threshold alternative models make an additional use of a noise parameter to accommodate data points that are literally incompatible with the model. To compute the posterior distribution over parameters and generate posterior predictive distributions, we ran for each model $4 \mathrm{MCMC}$ chains consisting of $10^{6}$ iterations discarding the first $5 \times 10^{5}$ for burn-in, and checked convergence qualitatively by visual inspection of the parameters and predictions of each chain. We additionally performed a formal Bayesian model comparison by computing the marginal likelihood of the data under each model (in order to compute Bayes Factors); to do this, for each model, we collected 1,000 samples from an Annealed Importance Sampling algorithm with an annealing schedule consisting of 50,000 uniformly spaced increments.

Overall, the uncertain threshold model is the best explanation of the distribution of responses observed in the ten experimental conditions (Table 5). For example, in the 0/25 distribution, both the model and participants tend to draw very weak interpretations $a$ la the Some model, whereas in the 25/100 distribution, the most dominant interpretation is more like All (Figure 12). The model is even able to accommodate subtle shifts in interpretation. For example, in the 25/75 distribution, most participants interpret the generic as implying roughly $75 \%$ of the category have the property, yet others (a minority) interpret the generic as implying only $25 \%$ have the property. This prediction is made by the uncertain threshold model: Higher prevalence levels are more likely under this model, but they are not the only interpretation of the generic; it is plausible that someone could utter this sentence with only in mind that a minority of the category have the property. The quantitative details of this kind of weak or inferior inference can also be observed by comparing the 25/100, 50/100, and 75/100 distributions, where there are two kinds of animals, those that always have the property and those that sometimes have the property (where what counts as "sometimes" varies between 25, 50, and 75\%). Here, the Uncertain threshold model predicts some subset of responses will be at the inferior mode $(25 / 50 / 75 \%)$ and that the proportion of responses at that inferior mode should increase as the mean of that mode increases $(25<50<75 \%)$, which is also observed in the empirical data. This uncertainty in the interpretation data is additionally a reason why the Reified kinds model falls short; this model always selects the component of the prior distribution with higher prevalence.

\section{General Discussion}

Generic language is the foremost case study of how abstract, generalizable knowledge is transmitted through language. Despite their ubiquity and relative morphosyntactic simplicity, generics have been examined by few quantitative studies and no quantitative models that make predictions about how generic language updates beliefs. Here, we explored the hypothesis proposed by Tessler and Goodman (2019) that generics update beliefs according to an uncertain threshold semantics. We tested these models using a diverse set of stimuli and showed that the generic interpretations can be predicted with high-quantitative accuracy as a function of background knowledge about the property updated by an uncertain threshold rule (Experiment 1). In Experiment 2, we showed how this model also explains differences observed by Cimpian et al. (2010). Furthermore, we found that the background 


\begin{tabular}{|c|c|c|c|c|c|}
\hline Model & Threshold & Noise & $r^{2}$ & MSE & (log) Bayes Factor \\
\hline Uncertain threshold & - & - & 0.948 & 0.0014 & 0 \\
\hline Prevalence Prior & - & - & 0.840 & 0.024 & -237 \\
\hline Some ( $0 \%$ threshold) & 0.01 & - & 0.941 & 0.0072 & -142 \\
\hline $10 \%$ threshold & 0.10 & 0.18 & 0.896 & 0.0089 & -165 \\
\hline $30 \%$ threshold & 0.30 & 0.37 & 0.844 & 0.0093 & -87 \\
\hline Most (50\% threshold) & 0.50 & 0.49 & 0.606 & 0.015 & -94 \\
\hline Best threshold & 0.73 & 0.68 & 0.312 & 0.022 & -51 \\
\hline Reified kinds & - & - & 0.948 & 0.0016 & -84 \\
\hline
\end{tabular}

Table 5

Summary statistics for models of Experiment 3. Mean Squared Errors and variance explained are calculated at the level of the distribution condition means. Bayes Factors quantify evidence in support of each model relative to the Uncertain threshold model and are computed over the full distribution of responses for Expt. 3 a 8 Expt. 3b. Numbers below 0 indicate evidence in support of the Uncertain threshold model.

knowledge about the expected prevalence of the feature was causally responsible for how novel generic statements are interpreted (Experiment 3). This work validates a computational formalism to articulate how beliefs are updated from generic language, which appears to be adequate within the domains considered; it represents a first step in understanding how generics serve to convey knowledge between people and generations.

The experimental data we present shows that generics can receive a systematic spectrum of prevalence-based interpretations, including being interpreted as only applying to a minority of the category (what we might call weak generics). Statements like Lorches live in zoos, Feps perform in the circus, and Wugs have seizures all received average implied prevalence ratings below $50 \%$, with many individual participants providing ratings of $10 \%$ or less (Expt. 1b). When manipulating the prevalence prior distributions to suggest only prevalence levels of around $0 \%$ or around $25 \%$, participants readily interpret the statement Ks know when earthquakes are about to happen to mean about a quarter of them do (Expt. $3 \mathrm{~b})$. These results highlight the variety of generic interpretations, which stand in contrast to a common view in the psychological literature on generics that generics (in a generic sense) convey monolithic, strong meanings. Here, we trace the variability of generic interpretations to more general prior beliefs, demonstrating how generics can convey strong implications, weak implications, and all shades in-between. ${ }^{17}$

\footnotetext{
${ }^{17}$ The response measure we use in our paradigm, as in the previous paradigms on generic interpretations, might encourage gradience even if gradience was limited. For example, Armstrong et al. (1983) showed that one can elicit gradience from objectively binary properties (e.g., the "evenness" of numbers). Gradience in property prevalence interpretations (e.g., how many wugs have seizures), which we examine in this paper, is distinct, however, from gradience in property knowledge (e.g., whether or not an individual instance or episode could be a gradient exemplar of a property such as a seizure). Our results cannot speak as to whether or not property knowledge per se is gradient, just that knowledge of prevalence is gradient. Prevalence knowledge being gradient is not particularly surprising given that the underlying construct is itself gradient (i.e., prevalence is a probability or frequency, which is a number between 0 - 1).
} 


\subsection{Generics, statistics, and concepts}

A dominant view in psychology is that the meaning of generic statements cannot be reduced to statistical information about the prevalence of the feature in the category (e.g., Leslie, 2008; Cimpian, Brandone, \& Gelman, 2010; Khemlani et al., 2012; Prasada et al., 2013). This conclusion is often arrived at by only considering fixed-threshold models of a prevalence-based semantics. Our comparisons to models that assume fixed-thresholds support this narrow conclusion, that a fixed-threshold semantics for generics is difficult to support given the data. The broader conclusion - rejecting all possible semantic models based on prevalence - is not warranted, however (see also Kochari et al., 2020 for a similar argument). The meanings of many expressions in natural language depend inextricably on context. For example, gradable adjectives like tall or expensive do not convey a single precise quantity of height or price (e.g., an expensive watch is probably a different price than an expensive hamburger), and yet we do not conclude from that the core meaning of tall has nothing to do with height or that the meaning of expensive is not really about the price of the object. The meanings of gradable adjectives are often arrived by considering a comparison class of other objects of the same type (e.g., an expensive hamburger is one that has a price that is high relative to other hamburgers; Kennedy, 2007) or which is pragmatically felicitous (Tessler, Lopez-Brau, \& Goodman, 2017), and the meaning of vague adjectives can be modeled using an uncertain threshold in a way completely analogous to how we have modeled the meaning of generics (Lassiter \& Goodman, 2017). Therefore, we conclude that quantitative, prevalence information can be at the core of generic meaning, but that such a meaning must be vague and context-sensitive (see also Sterken, 2015; Nickel, 2016 for related ideas about context-sensitivity of generics, and see van Rooij \& Schulz, 2020 for a mathematically similar account of generics).

At the same time, generics are known to influence conceptual knowledge. The fact that generics refer to abstractions has pinpointed them as potentially central to the growth of conceptual knowledge (Gelman, 2003, 2009), such as intuitive or folk theories of biology or psychology (Carey, 1985; Gopnik \& Meltzoff, 1997). Generics can also encode normative beliefs about objects or people (Göckeritz, Schmidt, \& Tomasello, 2014; Roberts, Ho, \& Gelman, 2017; Orvell, Kross, \& Gelman, 2018). In our model of generics, conceptual changes can occur at a higher level of abstraction above our minimalist, prevalence prior representations of world knowledge (e.g., the prevalence prior may be a lower-level variable in a rich, generative model of kinds and properties a la Kemp, Shafto, \& Tenenbaum, 2012). That is, the prevalence prior is an intermediate representation between conceptual knowledge (e.g., which could encode normative beliefs) and truth-functional semantics with a quantifiable, unified metric to evaluate whether a statement is true or false. Our model does take the strong view that the mechanism by which generics influence conceptual beliefs is via beliefs about prevalence or probability. This view may turn out to be incorrect (e.g., a notion of value could also be a part of the core meaning of generics, as formalized by van Rooij \& Schulz, 2020), but in our three heterogenous data sets that we have modeled and presented here, we do not see any gap between the uncertain threshold model predictions

and the empirical data, suggesting that prevalence alone in the semantics of generics is sufficient. 
It should be stressed that prevalence knowledge that we identify as central to generic language understanding does not need to come from observing frequencies in the world. Prevalence knowledge could itself be learned from language (e.g, through explanations; Lombrozo, 2006; Williams \& Lombrozo, 2010; Lombrozo \& Gwynne, 2014) and be informed by more general beliefs, such as intuitive theories about kinds of properties (cf., Keil, 1979; Nisbett et al., 1983). Formalizing the connections between general beliefs, other kinds of language, and generic language via prevalence priors is an important future direction for this line of work.

\subsection{Generics as a vehicle for cultural transmission}

The model we examined closely in this paper was first proposed as a listener model embedded inside of a pragmatic speaker model designed to predict generic endorsements, or truth judgments (Tessler \& Goodman, 2019). Tessler and Goodman (2019) showed that the generic endorsement judgments (e.g., do you endorse the statement: Mosquitos carry malaria?) can be predicted by this speaker model deciding whether or not to say the generic to a naive listener, where the naive listener updates its prior beliefs via an uncertain threshold function. We show here that the model of the naive listener - the Uncertain threshold model-perfectly tracks with the generic interpretation data (Expt. 1b, $2 \mathrm{~b}, 3 \mathrm{~b}$ ), in a manner that closely resembles how the predictions of the speaker model of Tessler and Goodman (2019) closely tracked with the variability among generic endorsements. Taken together, this pair of results - the speaker model of Tessler and Goodman (2019) predicts generic endorsements, and the listener model (about which the speaker model reasons) predicts generic interpretations - points to a more general conclusion that our unified, model-based approach warrants: Generic endorsements and interpretations behave as one would expect if speakers and listeners are rational users of language. More broadly, speakers and listeners use and interpret generics in a manner indicative of a mechanism for faithful transmission of knowledge (Tomasello, 1999). That is, at least for our set of stimuli, listeners interpret generics in the ways that speakers intend. This conclusion supports the role of generic language in the broader toolkit for human cultural transmission (see also Tomasello, 2016; Gelman \& Roberts, 2017).

There are some important features of our paradigm that make our experiments a kind of "best case" scenario for finding evidence for faithful knowledge transmission and should be taken into account to understand the generalizability of this conclusion. Foremost is that our stimuli are all generics about animals categories and properties, the statistics of which are relatively common knowledge and uncontroversial. For features that have difficult-to-understand statistical properties (e.g., features of social categories; Judd, Ryan, \& Park, 1991), prior beliefs about the prevalence of features can easily be misaligned between any individual pair of speaker and listener, potentially leading to the propagation of misinformation or stereotypes (see also Roberts et al., 2017). A second crucial feature of our designs that impact the generalizable of this conclusion is that our studies are all done with adults. The usage of generics as a mechanism of transmitting knowledge to children may also result in unfaithful transmission due to different prior beliefs throughout development (e.g., Brandone, 2017). Testing the Uncertain threshold model (and the speaker model component in Tessler \& Goodman, 2019) with generics about socially sensitive topics 
and in development are important future directions for this approach.

\subsection{The relationship between prevalence priors and generic interpretations}

In our experiments, we predict participants' interpretations of novel generics in terms of prevalence with our computational model as a function of empirical measurements of participants' background knowledge about properties, specifically background knowledge about the prevalence of properties. In Expt. 1a, we elicited background knowledge by having participants first generate different animal categories and then later rate prevalence for different properties of those animals. One might worry that there is a potential for circularity because we are predicting beliefs updated from language (generic interpretation; Expt. 1b) via beliefs elicited partly from language (prevalence prior; Expt. 1a). Perhaps participants answer the prevalence prior questions (e.g., What percentage of dogs develop phobias?) by first asking themselves internally a generic question (Do dogs develop phobias?) and then if the answer to this question was yes, responding with their personal (already generically-interpreted) prevalence (and rating 0\% prevalence if the answer to the generic question was no). This procedure would be consistent with a view of generics as representationally primary (as posited by Prasada, 2000 in the form of "generic beliefs") and in which prevalence estimates are derived by these "generic beliefs". Under this view, the two experiments would be measuring essentially the same construct, with the latter experiment (generic interpretation; Expt. 1b) restricting to kinds that "generically have the property".

Our formal model comparisons provide strong evidence against this interpretation. First, the Some model - which updates the prior by ruling out the $0 \%$ prevalence part of the distribution - is a simple control model that captures the reasoning articulated above: If there is an inherently binary kind of reasoning occurring ("kinds either do not do not [generically] have the property"), we would expect updating the prior by removing the $0 \%$ prevalence part of the distribution to return whatever it means to "generically have the property". The Some model consistently under-predicts the generic interpretation data, however, and was easily ruled out by model comparison given our data. An even more sophisticated version of this hypothesis is formalized by the Reified kinds model, which explicitly reasons about the prevalence in a kind of binary fashion: It looks at the prior as a mixture of two distributions (intuitively, the "haves" vs. the "have nots") and selects the distribution with the higher mean prevalence value (the "haves"). While the predictions of this model closely track the predictions of the Uncertain threshold model for many generics, the Reified kinds model over-predicts the prevalence ratings for generics that convey weak interpretations (e.g., Dorbs live in zoos) and is ruled out consistently by the data via formal model comparison. In addition, the Reified kinds model is unsatisfactory from a theoretical perspective because it ties the meaning of a generic to a particular structural assumption about world knowledge (e.g., the prevalence prior as a mixture distribution). These results support the idea that belief-updating from generics is more nuanced that simply fixing the binary value of a "generic belief" node to be true (cf., Prasada, 2000; Csibra and Shamsudheen, 2015). Instead, it is most parsimonious to conclude from these experiments that generics update prior beliefs about prevalence in a graded fashion, modeled as an uncertain threshold rule. 


\subsection{Generics and pragmatics}

The uncertain threshold model of generic interpretation that we test and validate in this paper is a kind of Literal Listener model used in probabilistic models of pragmatic language use (e.g., the Rational Speech Act theory; Frank \& Goodman, 2012; N. D. Goodman \& Frank, 2016; Scontras, Tessler, \& Franke, 2018). It is noteworthy that we are able to predict the inferences from generics in these experiments without having to articulate a full-blown model of pragmatic reasoning (i.e., a Pragmatic Listener model). In the course of this project, we formalized and tested two different versions of a pragmatic listener model. ${ }^{18}$ The predictions of these pragmatic listener models were only subtly different than the literal listener model and we found that the models provided comparable fits to the data in comparison to our literal listener model, but were penalized in the model comparison due to their additional flexibility in the kinds of data they could predict (i.e., the pragmatic models were more flexible due to their structure and an additional free parameter governing the rationality of the hypothetical speaker who produced the generic).

Our observation that the predictions of the pragmatic listener models and the literal listener models were only subtly different suggests that an experiment likely needs to be designed specifically to arbitrate between these hypotheses in order to distinguish them. Such a paradigm should probably move beyond the artificial, hypothetical situations studied here and in previous work on generic interpretations. For example, an interactive paradigm where a listener can understand that a speaker produced a generic as an intentional action may be more well suited to tap into the perceived intents of the interlocutors.

\subsection{Conclusion}

An easy way to acquire abstract knowledge is to understand a generic statement produced by a helpful interlocutor. A computational model that understands generics as operating as a vague quantifier is able to predict the large variability in generic interpretations. This work provides further support for a computational approach to generic language, opening the door to understanding more precisely how abstract knowledge is learned from language and conveyed through generations.

\section{Acknowledgements}

This work was supported in part by NSF Graduate Research Fellowship DGE-114747 and NSF SBE Postdoctoral Research Fellowship Grant No. 1911790 to MHT, a Sloan Research Fellowship, ONR grant N00014-13-1-0788, and DARPA grant FA8750-14-2-0009 to NDG, and Army Research Office MURI Grant No. W911NF-19-1-0057.

\footnotetext{
${ }^{18}$ Specifically, we formalized a model that treated the threshold variable as a "lifted" variable subject to recursive pragmatic reasoning (analogous to Lassiter and Goodman (2017)'s model gradable adjective interpretation) and another which marginalized out the threshold variable at the level of the literal listener before engaging in recursive, pragmatic reasoning.
} 


\section{References}

Abelson, R. P., \& Kanouse, D. E. (1966). Subjective acceptance of verbal generalizations. In S. Feldman (Ed.), Cognitive consistency: Motivational antecedents and behavioral consequents (p. 171-197). Academic Press.

Armstrong, S. L., Gleitman, L. R., \& Gleitman, H. (1983). What some concepts might not be. Cognition, 13(3), 263-308.

Brandone, A. C. (2017). Changes in beliefs about category homogeneity and variability across childhood. Child development, 88(3), 846-866.

Brandone, A. C., Gelman, S. A., \& Hedglen, J. (2014). Children's Developing Intuitions About the Truth Conditions and Implications of Novel Generics Versus Quantified Statements. Cognitive science, 1-28.

Carey, S. (1985). Conceptual change in childhood.

Carlson, G. N. (1977). Reference to kinds in english (Unpublished doctoral dissertation). University of Massachusetts, Amherst.

Cimpian, A., Brandone, A. C., \& Gelman, S. A. (2010). Generic statements require little evidence for acceptance but have powerful implications. Cognitive science, 34(8), $1452-1482$.

Cimpian, A., Gelman, S. A., \& Brandone, A. C. (2010). Theory-based considerations influence the interpretation of generic sentences. Language and Cognitive Processes, 25(2), 261-276. doi: 10.1080/01690960903025227.Theory-based

Cimpian, A., \& Markman, E. M. (2008). Preschool children's use of cues to generic meaning. Cognition, 107, 19-53. doi: 10.1016/j.cognition.2007.07.008

Cimpian, A., \& Markman, E. M. (2009). Information learned from generic language becomes central to children's biological concepts: Evidence from their open-ended explanations. Cognition, 113(1), 14-25. Retrieved from http://dx.doi.org/10.1016/j.cognition .2009.07.004 doi: 10.1016/j.cognition.2009.07.004

Cohen, A. (1999). Generics, Frequency Adverbs, and Probability. Linguistics and Philosophy, 22.

Csibra, G., \& Shamsudheen, R. (2015). Nonverbal Generics: Human Infants Interpret Objects as Symbols of Object Kinds. Annual Review of Psychology, 66, 689-710. doi: 10.1146/annurev-psych-010814-015232

Frank, M. C., \& Goodman, N. D. (2012). Predicting pragmatic reasoning in language games. Science, 336(6084).

Gelman, S. A. (1988). The development of induction within natural kind and artifact categories. Cognitive psychology, 20(1), 65-95.

Gelman, S. A. (2003). Essential child: Origins of essentialntialism in everyday thought. Oxford University Press.

Gelman, S. A. (2004). Learning words for kinds: Generic noun phrases in acquisition. In Weaving a lexicon (p. 445-484). MIT Press.

Gelman, S. A. (2009). Learning from others: Children's construction of concepts. Annual review of psychology, 60, 115-140. doi: 10.1146/annurev.psych.59.103006.093659 .LEARNING

Gelman, S. A., Coley, J. D., Rosengren, K. S., Hartman, E., \& Pappas, A. (1998). Beyond labeling: the role of maternal input in the acquisition of richly structured categories. 
Monographs of the Society for Research in Child Development, 63(1), I-V, 1-148; discussion 149-157.

Gelman, S. A., Goetz, P. J., Sarnecka, B. W., \& Flukes, J. (2008). Generic Language in Parent-Child Conversations. Language Learning and Development, 4(1), 1-31. doi: 10.1080/15475440701542625.Generic

Gelman, S. A., \& Roberts, S. O. (2017). How language shapes the cultural inheritance of categories. Proceedings of the National Academy of Sciences, 114(30), 7900-7907.

Gelman, S. A., Star, J. R., \& Flukes, J. E. (2002). Children's Use of Generics in Inductive Inferences. Journal of Cognition and Development, 3(2), 179-199.

Gelman, S. A., Taylor, M. G., Nguyen, S. P., Leaper, C., \& Bigler, R. S. (2004). Motherchild conversations about gender: Understanding the acquisition of essentialist beliefs. Monographs of the Society for Research in Child Development, 69(1), pp. i+v+vii+1142. Retrieved from http://www.jstor.org/stable/3701396

Gelman, S. A., Ware, E. A., \& Kleinberg, F. (2010). Effects of generic language on category content and structure. Cognitive Psychology, 61, 273-301. Retrieved from https://ac.els-cdn.com/S0010028510000344/ 1-s2.0-S0010028510000344-main.pdf?\{_\}tid=28ca76c8-b6ba-11e7-8a7a -00000 aacb35d $\{\&\}$ acdnat $=1508629812\left\{\_\right\} 86 c c 7468 \mathrm{a} 1 \mathrm{fdbd} 49 \mathrm{ac} 7 \mathrm{~d} 457 \mathrm{a} 5 \mathrm{c} 45 \mathrm{bead}$ doi: 10.1016/j.cogpsych.2010.06.001

Göckeritz, S., Schmidt, M. F., \& Tomasello, M. (2014). Young children's creation and transmission of social norms. Cognitive Development, 30, 81-95.

Goodman, N. (1955). Fact, fiction, and forecast. Harvard University Press.

Goodman, N. D., \& Frank, M. C. (2016). Pragmatic language interpretation as probabilistic inference. Trends in Cognitive Sciences.

Goodman, N. D., \& Stuhlmüller, A. (2013). Knowledge and implicature: Modeling language understanding as social cognition. Topics in Cognitive Science.

Goodman, N. D., \& Stuhlmüller, A. (2014). The Design and Implementation of Probabilistic Programming Languages. http://dippl.org. (Accessed: 2015-7-17)

Gopnik, A., \& Meltzoff, A. N. (1997). Words, thoughts, and theories. Mit Press.

Judd, C. M., Ryan, C. S., \& Park, B. (1991). Accuracy in the judgment of in-group and out-group variability. Journal of personality and social psychology, 61 (3), 366.

Keil, F. C. (1979). Semantic and conceptual development. Harvard University Press.

Kemp, C., Shafto, P., \& Tenenbaum, J. B. (2012). An integrated account of generalization across objects and features. Cognitive Psychology, 64(1-2), 35-73.

Kennedy, C. (2007). Vagueness and grammar: the semantics of relative and absolute gradable adjectives. Linguistics and Philosophy, 30, 1-35. doi: 10.1007/s10988-006-9008-0

Khemlani, S., Leslie, S.-J., \& Glucksberg, S. (2009). Generics, prevalence, and default inferences. In Proceedings of the 31st annual conference of the cogntiive science society. Austin, TX.

Khemlani, S., Leslie, S.-J., \& Glucksberg, S. (2012, July). Inferences about members of kinds: The generics hypothesis. Language and Cognitive Processes, 27(6), 887900. Retrieved from http://www.tandfonline.com/doi/abs/10.1080/01690965 .2011.601900 doi: 10.1080/01690965.2011.601900

Kochari, A., Van Rooij, R., \& Schulz, K. (2020). Generics and alternatives. Frontiers in Psychology, 11. 
Lassiter, D., \& Goodman, N. D. (2017). Adjectival vagueness in a bayesian model of interpretation. Synthese.

Lee, M. D., \& Wagenmakers, E. (2014). Bayesian cognitive modeling: A practical course. Cambridge: Cambridge University Press.

Leslie, S.-J. (2007). Generics and the Structure of the Mind. Philosophical Perspectives, 21(1), 375-403.

Leslie, S.-J. (2008, July). Generics: Cognition and acquisition. Philosophical Review, $117(1)$.

Lombrozo, T. (2006). The structure and function of explanations. Trends in cognitive sciences, $10(10), 464-470$.

Lombrozo, T., \& Gwynne, N. Z. (2014). Explanation and inference: Mechanistic and functional explanations guide property generalization. Frontiers in Human Neuroscience, 8,700 .

Montague, R. (1973). The Proper Treatment of Quantification in Ordinary English. In Philosophy, language, and artificial intelligence (pp. 141--162). Springer. Retrieved from http://semantics.uchicago.edu/kennedy/classes/s08/ semantics $2 /$ montague 73 .pdf

Neal, R. M. (2001). Annealed importance sampling. Statistics and computing, 11(2), $125-139$.

Nickel, B. (2016). Between logic and the world. Oxford: Oxford UP.

Nisbett, R. E., Krantz, D. H., Jepson, C., \& Kunda, Z. (1983). The use of statistical heuristics in everyday inductive reasoning. Psychological Review, 90(4), 339-363. doi: 10.1037/0033-295X.90.4.339

Orvell, A., Kross, E., \& Gelman, S. A. (2018). That's how "you" do it: Generic you expresses norms during early childhood. Journal of experimental child psychology, 165, 183-195.

Prasada, S. (2000). Acquiring generic knowledge. Trends in Cognitive Sciences, 4(2), 66-72. doi: $10.1016 /$ S1364-6613(99)01429-1

Prasada, S., \& Dillingham, E. M. (2006). Principled and statistical connections in common sense conception. Cognition, 99(1), 73-112. doi: 10.1016/j.cognition.2005.01.003

Prasada, S., Khemlani, S., Leslie, S.-J., \& Glucksberg, S. (2013, March). Conceptual distinctions amongst generics. Cognition, 126(3), 405-22. doi: 10.1016/j.cognition .2012 .11 .010

Rhodes, M., Leslie, S.-J., \& Tworek, C. M. (2012). Cultural transmission of social essentialism. Proceedings of the National Academy of Sciences, 109 (34), 13526-13531. doi: 10.1073/ pnas.1208951109

Ritchie, D., Stuhlmüller, A., \& Goodman, N. D. (2016). C3: Lightweight incrementalized mcmc for probabilistic programs using continuations and callsite caching. In Aistats 2016.

Roberts, S. O., Ho, A. K., \& Gelman, S. A. (2017). Group presence, category labels, and generic statements influence children to treat descriptive group regularities as prescriptive. Journal of Experimental Child Psychology, 158, 19-31.

Scontras, G., Tessler, M. H., \& Franke, M. (2018). Probabilistic language understanding: An introduction to the Rational Speech Act framework.

Shepard, R. N. (1987). Toward a universal law of generalization for psychological science. Science (New York, N.Y.), 237(4820), 1317-1323. Retrieved from http://gureckislab 
.org/courses/fall13/learnmem/papers/Shepard1987.pdfhttp://www.ncbi.nlm . nih.gov/sites/entrez?Db=pubmed $\{\&\}$ DbFrom=pubmed $\{\&\}$ Cmd $=\operatorname{Link}\{\&\}$ LinkName $=$ pubmed $\{$ \} pubmed $\{\&\}$ LinkReadableName $=$ RelatedArticles $\{\&\}$ IdsFromResult $=$ $3629243\{\&\}$ ordinalpos $=3\{\&\}$ itool=EntrezSystem2 doi: 10.1126/science. 3629243

Shmueli, G. (2010). To explain or to predict? Statistical science, 25(3), 289-310.

Sterken, R. K. (2015). Generics in Context. Philosophers' Imprint, 15(i), 1-30.

Tenenbaum, J. B., Kemp, C., Griffiths, T. L., \& Goodman, N. D. (2011, mar). How to grow a mind: statistics, structure, and abstraction. Science (New York, N.Y.), 331(6022), 1279-85. Retrieved from http://www.ncbi.nlm.nih.gov/pubmed/21393536 doi: $10.1126 /$ science. 1192788

Tessler, M. H., \& Goodman, N. D. (2019). The language of generalization. Psychological Review.

Tessler, M. H., Lopez-Brau, M., \& Goodman, N. D. (2017). Warm (for winter): Comparison class understanding in vague language. In Proceedings of the thirty-ninth annual conference of the cognitive science society.

Tomasello, M. (1999). The cultural origins of human cognition. Cambridge, MA: Harvard University Press.

Tomasello, M. (2016). Cultural learning redux. Child development, 87(3), 643-653.

van Rooij, R., \& Schulz, K. (2020). Generics and typicality: a bounded rationality approach. Linguistics and Philosophy, 43(1), 83-117.

Williams, J. J., \& Lombrozo, T. (2010). The role of explanation in discovery and generalization: Evidence from category learning. Cognitive science, 34(5), 776-806.

Wu, C. M., Meder, B., Filimon, F., \& Nelson, J. D. (2017). Asking better questions: How presentation formats influence information search. Journal of Experimental Psychology: Learning, Memory, and Cognition, 43(8), 1274.

Yarkoni, T., \& Westfall, J. (2017). Choosing prediction over explanation in psychology: Lessons from machine learning. Perspectives on Psychological Science, 12(6), 11001122. 


\section{Appendix: Items}

\begin{tabular}{|l|}
\hline Property \\
\hline are afraid of dogs \\
\hline are afraid of loud noises \\
\hline are intelligent \\
\hline attack hikers \\
\hline attract mates by secreting pheromones \\
\hline cannibalize each other \\
\hline capture other animals territory \\
\hline carry Lyme disease \\
\hline carry malaria \\
\hline carry out premeditated murder \\
\hline chase their tails \\
\hline develop back problems \\
\hline develop phobias \\
\hline do handstands to scare off predators \\
\hline drink alcohol left behind by tourists \\
\hline drink soda \\
\hline eat candy wrappers \\
\hline eat cannabis \\
\hline eat garbage \\
\hline eat grass \\
\hline eat human food \\
\hline eat insects \\
\hline eat people \\
\hline experience emotions \\
\hline experience empathy \\
\hline feed on the carcasses of dead animals \\
\hline fish in the Hudson River \\
\hline fly into building windows \\
\hline get addicted to nicotine \\
\hline get cancer \\
\hline get dandruff \\
\hline get erections \\
\hline get in fights with other animals \\
\hline give birth underwater \\
\hline go bald \\
\hline have a menstrual cycle \\
\hline have an exquisite sense of smell \\
\hline have brown fur \\
\hline have dozens of sexual partners \\
\hline have four legs \\
\hline have intensely beautiful feathers \\
\hline have personalities \\
\hline have seizures \\
\hline have spots \\
\hline have strange genetic mutations \\
\hline have very long wings \\
\hline
\end{tabular}


Table A1

\begin{tabular}{|l|}
\hline hunt other animals \\
\hline know how to open doors \\
\hline know how to ride bicycles \\
\hline know when earthquakes are about to happen \\
\hline lay eggs in other birds nests \\
\hline lay eggs without needing fertilization \\
\hline like to cuddle \\
\hline live in high-rise buildings \\
\hline live in the hulls of sea vessels \\
\hline live in trees \\
\hline live in urban areas \\
\hline live in zoos \\
\hline live to be a hundred years old \\
\hline live to be five hundred years old \\
\hline live to be twenty years old \\
\hline lose their teeth \\
\hline mourn their dead \\
\hline perform in the circus \\
\hline play with bottlecaps \\
\hline pound their chests to display dominance \\
\hline ride the subway \\
\hline sing beautiful songs \\
\hline sleep during the day \\
\hline steal farmers crops \\
\hline swim in shallow pools \\
\hline torture other animals \\
\hline transmit HIV \\
\hline transmit rabies \\
\hline use tools \\
\hline
\end{tabular}

Items used in Experiment 1

\begin{tabular}{|l||l|}
\hline Property & Property type \\
\hline broken legs & accidental \\
\hline burned skin & accidental \\
\hline cracked claws & accidental \\
\hline dusty skin & accidental \\
\hline fungus-covered fur & accidental \\
\hline infected ears & accidental \\
\hline itchy tails & accidental \\
\hline muddy feathers & accidental \\
\hline rotten teeth & accidental \\
\hline sore legs & accidental \\
\hline sore teeth & accidental \\
\hline swollen ears & accidental \\
\hline torn feathers & accidental \\
\hline torn tails & accidental \\
\hline wet fur & accidental \\
\hline
\end{tabular}




\begin{tabular}{|l||l|}
\hline worn-out claws & accidental \\
\hline blue claws & color \\
\hline orange ears & color \\
\hline orange tails & color \\
\hline pink teeth & color \\
\hline purple feathers & color \\
\hline silver legs & color \\
\hline violet skin & color \\
\hline yellow fur & color \\
\hline claws & part \\
\hline ears & part \\
\hline feathers & part \\
\hline fur & part \\
\hline legs & part \\
\hline skin & part \\
\hline tails & part \\
\hline teeth & part \\
\hline big claws & vague \\
\hline curly fur & vague \\
\hline long legs & vague \\
\hline long tails & vague \\
\hline long teeth & vague \\
\hline rough skin & vague \\
\hline small ears & vague \\
\hline smooth feathers & vague \\
\hline
\end{tabular}

Table A2

Items used in Experiment 2. All predicates were paired with the verb have/has. 